\title{
Recent Advances in Heat Transfer Enhancements: A Review Report
}

\author{
M. Siddique, A.-R. A. Khaled, N. I. Abdulhafiz, and A. Y. Boukhary \\ Thermal Engineering and Desalination Technology Department, King Abdulaziz University, \\ P.O. Box 80204 Jeddah 21589, Saudi Arabia \\ Correspondence should be addressed to A.-R. A. Khaled, akhaled4@yahoo.com
}

Received 25 March 2010; Revised 25 June 2010; Accepted 25 June 2010

Academic Editor: Alfons Baiker

Copyright ( $\odot 2010$ M. Siddique et al. This is an open access article distributed under the Creative Commons Attribution License, which permits unrestricted use, distribution, and reproduction in any medium, provided the original work is properly cited.

Different heat transfer enhancers are reviewed. They are (a) fins and microfins, (b) porous media, (c) large particles suspensions, (d) nanofluids, (e) phase-change devices, (f) flexible seals, (g) flexible complex seals, (h) vortex generators, (i) protrusions, and (j) ultra high thermal conductivity composite materials. Most of heat transfer augmentation methods presented in the literature that assists fins and microfins in enhancing heat transfer are reviewed. Among these are using joint-fins, fin roots, fin networks, biconvections, permeable fins, porous fins, capsulated liquid metal fins, and helical microfins. It is found that not much agreement exists between works of the different authors regarding single phase heat transfer augmented with microfins. However, too many works having sufficient agreements have been done in the case of two phase heat transfer augmented with microfins. With respect to nanofluids, there are still many conflicts among the published works about both heat transfer enhancement levels and the corresponding mechanisms of augmentations. The reasons beyond these conflicts are reviewed. In addition, this paper describes flow and heat transfer in porous media as a well-modeled passive enhancement method. It is found that there are very few works which dealt with heat transfer enhancements using systems supported with flexible/flexible-complex seals. Eventually, many recent works related to passive augmentations of heat transfer using vortex generators, protrusions, and ultra high thermal conductivity composite material are reviewed. Finally, theoretical enhancement factors along with many heat transfer correlations are presented in this paper for each enhancer.

\section{Introduction}

The way to improve heat transfer performance is referred to as heat transfer enhancement (or augmentation or intensification). Nowadays, a significant number of thermal engineering researchers are seeking for new enhancing heat transfer methods between surfaces and the surrounding fluid. Due to this fact, Bergles [1,2] classified the mechanisms of enhancing heat transfer as active or passive methods. Those which require external power to maintain the enhancement mechanism are named active methods. Examples of active enhancement methods are well stirring the fluid or vibrating the surface [3]. Hagge and Junkhan [4] described various active mechanical enhancing methods that can be used to enhance heat transfer. On the other hand, the passive enhancement methods are those which do not require external power to sustain the enhancements' characteristics. Examples of passive enhancing methods are: (a) treated surfaces, (b) rough surfaces, (c) extended surfaces, (d) displaced enhancement devices, (e) swirl flow devices, (f) coiled tubes, (g) surface tension devices, (h) additives for fluids, and many others.

\section{Mechanisms of Augmentation of Heat Transfer}

To the best knowledge of the authors, the mechanisms of heat transfer enhancement can be at least one of the following.

(1) Use of a secondary heat transfer surface.

(2) Disruption of the unenhanced fluid velocity.

(3) Disruption of the laminar sublayer in the turbulent boundary layer. 
(4) Introducing secondary flows.

(5) Promoting boundary-layer separation.

(6) Promoting flow attachment/reattachment.

(7) Enhancing effective thermal conductivity of the fluid under static conditions.

(8) Enhancing effective thermal conductivity of the fluid under dynamic conditions.

(9) Delaying the boundary layer development.

(10) Thermal dispersion.

(11) Increasing the order of the fluid molecules.

(12) Redistribution of the flow.

(13) Modification of radiative property of the convective medium.

(14) Increasing the difference between the surface and fluid temperatures.

(15) Increasing fluid flow rate passively.

(16) Increasing the thermal conductivity of the solid phase using special nanotechnology fabrications.

Methods using mechanisms no. (1) and no. (2) include increasing the surface area in contact with the fluid to be heated or cooled by using fins, intentionally promoting turbulence in the wall zone employing surface roughness and tall/short fins, and inducing secondary flows by creating swirl flow through the use of helical/spiral fin geometry and twisted tapes. This tends to increase the effective flow length of the fluid through the tube, which increases heat transfer but also the pressure drop. For internal helical fins however, the effect of swirl tends to decrease or vanish all together at higher helix angles since the fluid flow then simply passes axially over the fins [5]. On the other hand, for twisted tape inserts, the main contribution to the heat transfer augmentation is due to the effect of the induced swirl. Due to the form drag and increased turbulence caused by the disruption, the pressure drop with flow inside an enhanced tube always exceeds that obtained with a plain tube for the same length, flow rate, and diameter.

Turbulent flow in a tube exhibits a low-velocity flow region immediately adjacent to the wall, known as the laminar sublayer, with velocity approaching zero at the wall. Most of the thermal resistance occurs in this lowvelocity region. Any roughness or enhancement technique that disturbs the laminar sublayer will enhance the heat transfer [6]. For example, in a smooth tube of $25.4 \mathrm{~mm}$ inside diameter, at $\mathrm{Re}=30,000$, the laminar sublayer thickness is only $0.0762 \mathrm{~mm}$ under fully developed flow conditions. The internal roughness of the tube surface is well-known to increase the turbulent heat transfer coefficient. Therefore, for the example at hand, an enhancement technique employing a roughness or fin element of height $\sim 0.07 \mathrm{~mm}$ will disrupt the laminar sublayer and will thus enhance the heat transfer. Accordingly, mechanism no. (3) is a particularly important heat transfer mechanism for augmenting heat transfer.

Li et al. [5] described the flow structure in helically finned tubes using flow visualization by means of high-speed photography employing the hydrogen bubble technique. They used four tubes with rounded ribs having helix angles between $38^{\circ}$ and $80^{\circ}$ and one or three fin starts, in their investigation. Photographs taken by them showed that in laminar flow, bubbles follow parabolic patterns whereas in the turbulent flow, these patterns break down because of random separation vortices. Also, for tubes with helical ridges, transition to turbulent flow was observed at lower Reynolds numbers compared to smooth tube values. Although swirl flow was observed for all tubes in the turbulent flow regime, the effect of the swirl was observed to decrease at higher helix angles. Li et al. [5] concluded that spiral flow and boundary-layer separation flow both occurred in helicalridging tubes, but with different intensities in tubes having different configurations. As such, mechanisms no. (4) and no. (5) are also important heat transfer mechanisms for augmenting heat transfer.

Arman and Rabas [7] discussed the turbulent flow structure as the flow passes over a two-dimensional transverse rib. They identified the various flow separation and reattachment/redevelopment regions as: (a) a small recirculation region in front of the rib, (b) a recirculation region after the rib, (c) a boundary layer reattachment/redevelopment region on the downstream surface, and finally (d) flow up and over the subsequent rib. The authors noting that recirculation eddies are formed above these flow regions, identified two peaks that occur in the local heat transfer-one at the top of the rib and the other in the downstream recirculation zone just before the reattachment point. They also stated that heat transfer enhancement increases substantially with increasing Prandtl number. Therefore, the mechanism no. (6) plays an important role in heat transfer enhancements.

Heat transfer enhancements associated with fully/partially filing the fluidic volume by the porous medium take place by the following mechanisms $[8,9]$.

(7) Enhancing effective thermal conductivity of the fluid under static conditions.

(8) Enhancing effective thermal conductivity of the fluid under dynamic conditions.

(9) Delaying the boundary layer development.

(10) Thermal dispersion.

(11) Increasing the order of the fluid molecules.

(12) Redistribution of the flow.

(13) Modification of radiative property of the convective medium.

Ding et al. [10] showed that fluids containing $0.5 \mathrm{wt} . \%$ of carbon nanotubes (CNT) can produce heat transfer enhancements over $250 \%$ at $\mathrm{Re}=800$, and the maximum enhancement occurs at an axial distance of approximately 110 times the tube diameter. These types of mixtures are named in the literature as "nanofluids" and it will discussed later on in this report. The increases in heat transfer due to presence of nanofluids are thought to be associated with the following mechanisms.

(7) Enhancing effective thermal conductivity of the fluid under static conditions. 
(8) Enhancing effective thermal conductivity of the fluid under dynamic conditions.

(9) Delaying the boundary layer development.

(10) Thermal dispersion.

(11) Increasing the order of the fluid molecules.

(12) Redistribution of the flow.

Flexible fluidic thin films were introduced in the work of Khaled and Vafai [11, 12] and Khaled [13]. In their works, they describe a new passive method for enhancing the cooling capability of fluidic thin films. In summary, flexible thin films utilize soft seals to separate between their plates instead of having rigid thin film construction. Khaled and Vafai [11] have demonstrated that more cooling is achievable when flexible fluidic thin films are utilized. The expansion of the flexible thin film including flexible microchannel heat sink is directly related to the average internal pressure inside the microchannel. Additional increase in the pressure drop across the flexible microchannl not only increases the average velocity but also, it expands the microchannel causing an apparent increase in the coolant flow rate which, in turn, increases the cooling capacity of the thin film. Khaled and Vafai [12] and Khaled [13] have demonstrated that the cooling effect of flexible thin films can be further enhanced if the supporting soft seals contain closed cavities filled with a gas which is in contact with the heated plate boundary of the thin film. They referred to this kind of sealing assembly as "flexible complex seals". The resulting fluidic thin film device is expandable according to an increase in the working internal pressure or an increase in the heated plate temperature. Therefore, mechanism no. (15) can have an impact in enhancing heat transfer inside thermal systems. Finally, the mechanism no. (14) finds its applications when rooted fins are utilized as an enhancer of heat transfer [14]. Finally, the last mechanism will be discussed when the topic of ultra high thermal conductivity composite materials is discussed.

\section{Heat Transfer Enhancers}

From the concise summary about mechanisms of enhancing heat transfer described in last section, it can be concluded that these mechanisms can not be achieved without the presence of the enhancing elements. These elements will be called as "heat transfer enhancers". In this report, the following heat transfer enhancers will be explained: 3.1. Extended surfaces (Fins); 3.2. Porous media; 3.3. Large particles suspensions; 3.4. Nanofluids; 3.5. Phase change devices; 3.6. Flexible seals; 3.7. Flexible complex seals; 3.8. Vortex generators; 3.9. Protrusions; and 3.10. Ultra high thermal conductivity composite materials.

\subsection{Heat Transfer Enhancement Using Extended Surfaces (Fins)}

3.1.1. Introduction. Fins are quite often found in industry, especially in heat exchanger industry as in finned tubes of double-pipe, shell-and-tube and compact heat exchangers [15-20]. As an example, fins are used in air cooled finned tube heat exchangers like car radiators and heat rejection devices. Also, they are used in refrigeration systems and in condensing central heating exchangers. Moreover, fins are also utilized in cooling of large heat flux electronic devices as well as in cooling of gas turbine blades [21]. Fins are also used in thermal storage heat exchanger systems including phase change materials [22-25]. To the best knowledge of the authors, fins as passive elements for enhancing heat transfer rates are classified according to the following criteria.

(1) Geometrical design of the fin.

(2) Fins arrangements.

(3) Number of fluidic reservoirs interacting with the fin.

(4) Location of the fin base with respect to the solid boundary.

(5) Composition of the fin.

According to design aspects, fins can have simple designs, such as rectangular, triangular, parabolic, annular, and pin rod fins. On the other hand, fin design can be complicated such as spiral fins $[26,27]$. In addition, fins can have simple network as in finned tubes heat exchangers [20]. In contrast, they can be arranged in a complex network as can be seen in the works of [28-30]. Moreover, fins can be further classified based on the fact whether they interact thermally with a single fluid reservoir or with two different fluid reservoirs. Example of works based on the last classification is the works of Khaled [31, 32]. In addition, fins can be attached to the surface as in the works [15-25] or they may have roots in the heated/cooled walls [13]. Finally, fins can be solid $[3,4,15-25]$ or they can be porous [33] or permeable [34].

3.1.2. Laminar Single-Phase Heat Transfer in Finned Tubes. Laminar flow generally results in low heat-transfer coefficients and the fluid velocity and temperature vary across the entire flow channel width so that the thermal resistance is not just in the region near the wall as in turbulent flow. Hence, small-scale surface roughness is not effective in enhancing heat transfer in laminar flow; the enhancement techniques employ some method of swirling the flow or creating turbulence [6]. Laminar flow heat transfer and pressure drop in "microfin" tubes (discussed later) was experimentally measured by [35]. Their data showed that the heat transfer and pressure drop in microfin tubes were just slightly higher than in plain tubes and they recommended that microfin tubes not be used for laminar flow conditions. This outcome has also been confirmed in the investigations of Shome and Jensen [36] who concluded that "microfinned tube and tubes with fewer numbers of tall fins are ineffective in laminar flows with moderate free convection, variable viscosity, and entrance effects as they result in little or no heat transfer enhancement at the expense of fairly large pressure drop penalty".

3.1.3. Turbulent Single-Phase Heat Transfer in Finned Tubes. Turbulent flow and heat transfer in finned tubes has been 
widely studied in the past and the literature available on the experimental investigations of turbulent flow and heat transfer in finned tubes is quite extensive. One of the earliest experimental work on the heat transfer and pressure drop characteristics of single-phase flows in internally finned tubes dates back to 1964 when Hilding and Coogan [37] presented their data for ten different internal fin geometries for a $0.55 \mathrm{in} .(14 \mathrm{~mm})$ inner diameter copper tube with $0.01 \mathrm{in} .(0.254 \mathrm{~mm})$ straight brass fins using air as the test fluid. Hilding and Coogan [37] observed that the heat transfer is enhanced by around $100-200 \%$ over that of the smooth tube and the enhancement is accompanied by a similar increase in the pressure drop. The Reynolds number in this study ranged from 15,00 to 50,000.

Kalinin and Yarkho [38] used different fluids in the range $1500 \leq \operatorname{Re} \leq 400,000$ and $7 \leq \operatorname{Pr} \leq 50$ to investigate the effect of Reynolds and Prandtl numbers on the effectiveness of heat transfer enhancement in smoothly outlined internally grooved tubes. The ranges of the transverse groove heights tested were $0.983 \leq d / D \leq 0.875$, (where $d$ is the fin tip diameter and $D$ is the inner diameter) with maximum groove spacing equal to the pipe nominal diameter. They reported that the critical Reynolds number at which transition to turbulent flow occurs decreased from 2400 for a smooth tube to 1580 at $e / D=0.875$ and fin spacing equal to half the pipe diameter for a grooved tube, with a maximum increase in the heat transfer coefficient of up to 2.2 times the minimum measured value. The authors also observed that the behavior of the Nusselt number for the tested range of Prandtl numbers is independent of the Prandtl number.

In their two papers, Vasilchenko and Barbaritskaya [39, 40] published their results for the heat transfer and pressure drop of turbulent oil flow in straight finned tubes with $4 \leq N \leq 8$ and $0.13 \leq e / D \leq 0.3$, for an operating condition range of $10^{3} \leq \operatorname{Re} \leq 10^{4}$ and $70 \leq \operatorname{Pr} \leq 140$. Their results showed that the heat transfer is enhanced by $30 \%$ to $70 \%$ over that of smooth tubes for the finned tubes tested. Correlations for predicting the friction factors and the Nusselt numbers were also presented.

In the work of Bergles et al. [41], heat transfer and pressure drop data for straight and spiral finned tubes of fin heights from 0.77 to $3.3 \mathrm{~mm}$ with water as the working fluid was investigated. The Reynolds number based on the hydraulic diameter ranged from around 1,500 to 50,000 . They found an earlier transition from laminar to turbulent flow and their friction factor data indicated that the smooth tube friction factor correlations could also be used for the tested finned tubes in the turbulent region. The heat transfer coefficients were found to be up to twice that of comparable smooth tubes. From their heat transfer data, they concluded that the hydraulic diameter approach is effective for correlation only in the case of straight fins of moderate heights.

Watkinson et al. $[42,43]$ in their two separate works, performed experiments for water and air flows, respectively, in a tube-in-tube heat exchanger under isothermal heating conditions to study the turbulent heat transfer and pressure drop characteristics of straight and helically finned tubes. A total of eighteen tubes, 5 with straight fins and 13 with spiral fins, having internal fin geometries with fin starts from 6 to $50,0.026 \leq e / D \leq 0.158$, helix angles from $0^{\circ}$ to $15^{\circ}$, and inside diameters of 0.420 to 1.196 inch were examined for $7 \times 10^{3} \leq \operatorname{Re} \leq 3 \times 10^{5}$ and $0.7 \leq \operatorname{Pr} \leq 3.4$. A commercial smooth copper tube was also tested for comparison. The air results presented show that for most tubes, from $\mathrm{Re}=50,000$ up to $\mathrm{Re}=300,000$ the heat transfer is enhanced up to $95 \%$ over that of a smooth tube. On the other hand, in water flow tests, at $\operatorname{Re}=50,000$ heat transfer is enhanced up to $87 \%$ over that of a smooth tube, but at higher Reynolds number, the finned tubes approached smooth tube heat transfer performance. A maximum increase of $100 \%$ in the pressure drop over smooth tube was observed for tubes with tall helical fins. Separate empirical nondimensional heat transfer correlations were presented for water and air, for both straight and spiral fin tubes, having a form similar to the smooth tube turbulent Sieder-Tate correlation with additional parameters consisting of the ratios of the inter fin spacing to the tube diameter and the fin pitch to the tube diameter. For straight fin tubes, the inter fin spacingto-diameter ratio and for spiral finned tubes the pitch-todiameter ratio were incorporated to form a modified Blasiustype correlation for predicting the friction factor. These correlations predicted their data to within a maximum error of $13 \%$.

Carnavos [44] tested eight finned tubes (both straight and helically finned) to obtain heat transfer and pressure drop data for cooling of air in turbulent flow employing a double tube heat exchanger. The tubes tested had fin starts from 6 to 16 and helix angles from $2.5^{\circ}$ to $20^{\circ}$. The results were presented on the hydraulic diameter basis for the range $10^{4} \leq \mathrm{Re}_{h} \leq 10^{5}$ and correlations were proposed to predict the heat transfer and the pressure drop. The reported heat transfer correlation was in the form of a modified Dittus-Boelter single-phase correlation having an additional correction factor " $F$ ", consisting of the ratios of the nominal heat transfer area to the actual heat transfer area and the actual flow area to the core flow area, respectively. The secant of the helix angle raised to the third power was also included in " $F$ ". The friction factor was also in the form of a modified Blasius type equation with a correction factor " $F^{*}$ " comprising of the ratio of the actual flow area-to-the nominal flow area and the secant of the helix angle. In a later investigation, Carnavos [45] used the same apparatus and three more tubes with number of fin starts and helix angles up to $38^{\circ}$ and $30^{\circ}$, respectively, to extend his air results by including experimental data conducted for heating of water and a $50 \% \mathrm{w} / \mathrm{w}$ ethylene glycol-water solution. The data for air obtained earlier [44] was reexamined and a set of correlations that predicted their entire data obtained with air, water, and ethylene glycol-water solution to within $\pm 10 \%$, were proposed in the ranges $10^{4} \leq \mathrm{Re}_{h} \leq 10^{5}, 0.7 \leq \mathrm{Pr} \leq$ 30 , and $0 \leq \alpha \leq 30^{\circ}$. The Nusselt number correlation was allowed to retain its original form whereas the helix angle dependency in the friction factor correlation was slightly changed.

Armstrong and Bergles [46] conducted experiments for electric heating of air in the range 9,000 $\leq \mathrm{Re} \leq 120,000$ and $\operatorname{Pr}=0.71$, using seven different silicon carbide finned 
tubes all having straight fins. The tubes tested had fin starts from 8 to 24 and $e / D$ from 0.06 to 0.15 . The results indicated that the heat transfer is enhanced by around $30-100 \%$ over that of a smooth silicon carbide tube. Their heat transfer data was predicted to within $\pm 20 \%$ by the Carnavos [45] heat transfer correlation, but a large disagreement was observed between the measured friction factors and those predicted by the Carnavos [45] friction factor correlation.

Since their introduction more than 20 years ago, "microfin" tubes have received a lot of attention playing a very significant role in modern, high-efficiency heat transfer systems. Microfin enhancements are of special interest because the amount of extra material required for microfin tubing is much less than that required for other types of internally finned tubes [47]. Of the many enhancement techniques which have been proposed, passively enhanced tubes are relatively easy to manufacture, cost-effective for many applications, and can be used for retrofitting existing units, whereas active methods, such as vibrating tubes, are costly and complex [48]. Moreover, these tubes ensure a large heat transfer enhancement with a relatively small increase in the pressure drop penalty. Microfin tubes are typically made of copper and have an outside diameter between 4 and $15 \mathrm{~mm}$. The principal geometric parameters that characterize these tubes [49] are: the external diameter, fin height (from $0.075 \mathrm{~mm}$ to $0.4 \mathrm{~mm}$ ), helix angle (from $10^{\circ}$ to $35^{\circ}$ ), and the number of fin starts (from $50^{\circ}$ to $60^{\circ}$ ). These dimensions are in contrast to other types of internal finning that seldom exceed 30 fins per inch and fin heights that range from several factors higher than the microfin tube height. Currently, tubes with axial and helical fins, in rectangular, triangular, trapezoidal, crosshatched, and herringbone patterns are available. Important dimensionless geometric variables of an internally microfinned tubes include the dimensionless fin height $(\varepsilon / D$, fin height/internal diameter) and the dimensionless fin pitch $(p / \varepsilon$, fin spacing/fin height). A microfin tube typically has $0.02 \leq \varepsilon / D \leq 0.04$ and $1.5 \leq p / D \leq 2.5$, [50]. As microfinnned tubes are typically used in evaporators and condensers, thus most of the extensive existing research literature on microfinned tube performance characteristics is devoted to two-phase refrigerant flows. Schlager et al. [51] and Khanpara et al. [52] are typical examples of such investigations, showing a $50 \%$ to $100 \%$ increase in boiling and condensation heat transfer coefficients with only a $20 \%$ to $50 \%$ increase in pressure drop. However, single-phase performance of microfinned tubes is also an important consideration in the design of refrigeration condensers as a substantial proportion of the heat transfer area of these condensers is taken up in the desuperheating and later subcooling of the refrigerant. Consequently, accurate correlations for predicting the single-phase heat transfer and pressure drop inside microfinned tubes are necessary in order to predict the performance of these condensers and to optimize the design of the system.

Khanpara et al. [52] investigated the heat transfer characteristics of R-113, testing eight microfinned tubes in the range $60 \leq N \leq 70,0.005 \leq e / D \leq 0.02$, and helix angles from $8^{\circ}$ to $25^{\circ}$, for $5 \times 10^{3} \leq \operatorname{Re} \leq 11 \times 10^{3}$. The results presented for the single phase heating of R-113 indicated that the heat transfer is enhanced by around $30 \%-80 \%$. The authors concluded that a major part of the enhancement is due to the increase in the area available for heat transfer and a part of the enhancement is due to flow separation and flow swirling effects induced by the helical fins. This is because the corresponding increase in the heat transfer area over that in a smooth tube is around $10 \%-50 \%$ for the tubes tested. In a subsequent paper, Khanpara et al. [53] also reported the local heat transfer coefficients for single-phase liquid R-22 and R-113 flowing through a smooth and an internally finned tube of $9.52 \mathrm{~mm}$ outer diameter, in their paper on in-tube evaporation and condensation characteristics of microfinned tubes. The single-phase experiments were performed by direct electrical heating of the tube walls in the Reynolds number range of 5,000 to 11,000 for R-113 and 21,000 to 41,000 for R-22. The microfinned tube had of 60 fin starts, a fin height of $0.22 \mathrm{~mm}$, and a helix angle of approximately $17^{\circ}$. The heat transfer coefficients for the internally finned tube were found to be $50 \%$ to a $150 \%$ higher than the smooth tube values.

AI-Fahed et al. [54] experimentally tested a single microfinned tube with a $15.9 \mathrm{~mm}$ outside diameter having 70 helical fins with fin height of $0.3 \mathrm{~mm}$ and a helix angle of $18^{\circ}$ using water as the test fluid in a tube-in-tube heat exchanger. Results were presented for isothermal heating conditions in the range $10,000<\operatorname{Re}<30,000$. Under the same conditions, comparative experiments with an internally smooth tube were also conducted. They noted that the heat transfer is enhanced by $20 \%-80 \%$ and the pressure drop is increased by around $30 \%-80 \%$ as compared to the smooth tube values. The experimentally obtained friction and heat transfer data were correlated as a Blasius and a Sieder-Tate type correlation, respectively. The heat transfer correlation predicted their data to within $\pm 25 \%$, showing a large error band while no error band was reported for the friction factor results. The authors reasoned that at $\operatorname{Re}>25,000$ the heat transfer enhancement ratio is moderate plausibly because at higher Re numbers the turbulence effect in microfinned tubes becomes similar to that in a plain tube.

Chiou et al. [55] conducted an experimental study with water using two internally finned tubes having the same outer diameter equal to $0.375 \mathrm{in}$. $(9.52 \mathrm{~mm})$. The two tubes had 60 and 65 fins, fin heights of 0.008 in $(0.20 \mathrm{~mm})$ and $0.01 \mathrm{in} .(0.25 \mathrm{~mm})$, and helix angles of 18 and $25^{\circ}$, respectively. The Reynolds number in this study ranged from about 4,000 to 30,000. Modified Dittus-Boelter type correlations were formulated to predict the value of the heat transfer coefficient for flow Reynolds number greater than about 15000 and 13000, respectively, for each tube. An available heat-momentum analogy based correlation for rough tubes along with a set of constitutive equations for calculating related roughness parameters was utilized to propose correlations for predicting the friction factor and the Nussult number for the entire range of the Reynolds number tested.

Brognaux et al. [50] obtained experimental single-phase heat transfer coefficients and friction factors for three singlegrooved and three cross-grooved microfin tubes all having an equivalent diameter of $14.57 \mathrm{~mm}$, a fin height of $0.35 \mathrm{~mm}$, 
and 78 helical fins; only the fin helix angle was allowed to vary between $17.5^{\circ}$ and $27^{\circ}$ Using liquid water and air as the test fluid the experiments were carried out in a double-pipe heat exchanger. Results were presented for cooling conditions in the range $2500<\operatorname{Re}<50,000$ and $0.7<\operatorname{Pr}<7.85$. Validation experiments with an internally smooth tube were also conducted using water and air. Compared to a smooth tube, the maximum heat transfer enhancement reported was $95 \%$ with a pressure drop increase of $80 \%$ for water at $\operatorname{Pr}=6.8$. They also found that the friction factors in microfin tubes do not reach a constant value at high Reynolds numbers as is usually observed in rough pipes. The authors also used their data in the range $0.7<\operatorname{Pr}<7.85$ (using only 2 of the tested tubes) to analyze the dependence of the heat transfer on the Prandtl number exponent. Using the heatmomentum transfer analogy as applied for rough surfaces, they presented their experimental heat transfer and friction factor data as functions of the "roughness Reynolds number" and from cross-plots deduced the Prandtl number exponent to be between 0.56-0.57. The Prandtl number exponent between $0.55-0.57$ was also determined for the power law formulation $\mathrm{Nu}=C \operatorname{Re}^{m} \operatorname{Pr}^{n}$. The authors also defined an "efficiency index" (which gives the ratio of the increase in heat transfer to the increase in friction factor for a finned and plain tube, resp.) and presented its value for the different tubes tested. The higher the efficiency index, the better the enhancement geometry.

Huq et al. [56] presented experimental heat transfer and friction data for turbulent air flow in a tube having internal fins in the entrance region as well as in the fully-developed region. The tube/fin assembly was cast from aluminum to avoid any thermal contact resistance. The uniformly heated test section was $15.2 \mathrm{~m}$ in length and the inner diameter of the tube was $70 \mathrm{~mm}$ which contained six equally spaced fins of height $15 \mathrm{~mm}$. The Reynolds number based on hydraulic diameter ranged from $2.6 \times 10^{4}$ to $7.9 \times 10^{4}$. The results presented by the authors exhibited high pressure gradients and high heat transfer coefficients in the entrance region, approaching the fully developed asymptotic values away from the entrance section. The enhancement of heat transfer rate due to integral fins was reported to be very significant over the entire range of flow rates studied in this experiment. Heat transfer coefficient, based on inside diameter and nominal area of finned tube exceeded unfinned tube values by $97 \%$ to $112 \%$ for the tested Reynolds number range. When compared at constant pumping power, an improvement as high as 52\% was also observed for the overall heat transfer rate.

With the expressed objective of developing physically based generally applicable correlations for Nusselt number and friction factor for the finned tube geometry, Jensen and Vlakancic [57] carried out a detailed experimental investigation of turbulent fluid flow in internally finned tubes covering a wide range of fin geometric and operating conditions. Two geometrically identical double pipe heat exchangers were used. The test fluid (water and ethylene glycol were used) flowed through the tube side of each of the heat exchangers in counter-flow with hot water in one test section and cold water in the other. Friction factor tests were also conducted under isothermal conditions. A total of sixteen pairs of tubes (15 finned and one smooth tube) with a wide range of geometric variations (inside diameter $24.64-21.18 \mathrm{~mm}$, helix angles $0^{\circ}-45^{\circ}$, fin height $0.18 \mathrm{~mm}-2.06 \mathrm{~mm}$, and number of fins $8-54$ ) were tested. In the reported results, the authors first described the parametric effects of different fin geometries on turbulent friction factors and Nusselt numbers in internally finned tubes and then go on to prescribe a criterion for labeling a tube as a "high" fin tube $(2 e / D>0.06)$ or a "micro" fin tube. They stated that a microfin tube is characterized by its peculiar pressure drop behavior with long lasting transitional flow up to $\operatorname{Re}=20,000$. Trends in the reported data are different depending on whether the tube is a high-fin or a microfin tube. High fin tubes show friction factors curves similar to those of a smooth tube, only displaced higher with the friction factor increasing as the number of fins increases. For microfin tubes, in general, the friction factor is insensitive to the fin height and the Reynolds number up to $\operatorname{Re}=20,000$, but beyond this value the friction factor showed a decreasing trend with increasing $R e$ as in smooth tubes and the effect of number of fins, fin height, and the helix angle also comes into play, whenever anyone of these parameter is increased the friction factor increased (exceptions may occur due to difference in fin profile). Overall the reported increase in friction factor for the high finned tubes ranged from $40 \%-170 \%$ and in microfin tubes from $40 \%-140 \%$, over smooth tubes. For both types of tubes the reported trends of the slope of the $\mathrm{Nu}$ curves generally followed that of the smooth tube; however, the trends revealed a different slope at lower Re for the two categories of tubes. This characteristic was attributed by the authors to the greater capacity of swirling flow for higher finned tubes. However, the trends with geometry were similar to those noted for the friction factors. Overall, the reported increase in $\mathrm{Nu}$ for the high finned tubes ranged from 50\%-150\% and in microfin tubes from $20 \%-220 \%$ over smooth tubes. They reported that the correlations from the literature poorly predict their data and based on the findings from the trends observed went on to develop new correlations for friction factors and Nusselt numbers separately for the two categories of tube categories (high and microfin) identified by them. These correlations are applicable to a wide range of geometric and flow conditions for both categories of tubes and estimated well their data as well as the data from the literature.

Webb et al. [58] investigated the heat transfer and fluid flow characteristics of internally helical ribbed tubes. Using liquid water as the test fluid the experiments were carried out in a double-pipe heat exchanger. Results were presented for cooling conditions in the range $20,000<\operatorname{Re}<80,000$ and $5.08<\operatorname{Pr}<6.29$. A total of eight tubes $(7$ ribbed and one smooth tube) all having an inside diameter of $15.54 \mathrm{~mm}$ but a wide range of geometric variations (helix angles $25^{\circ}-45^{\circ}$, rib height $0.327^{\circ} \mathrm{mm}-0.554 \mathrm{~mm}$, and number of fin starts 10-45) were tested. The authors presented power law based empirical correlations using their experimental data for the Colburn $j$-factor and the fanning friction factor, which predicted their data reasonably well. The finned tube 
performance efficiency index as defined by Brognaux et al. [50] was also determined for the tubes tested, from which the authors concluded that the two key factors that affect the increase of the heat transfer coefficient in helically-ribbed tubes are the area increase and fluid mixing in the inter fin region caused by flow separation and reattachment, and the combination of the two determines the level of the heat transfer enhancement.

Copetti et al. [49] tested a single internally microfinned tube of $9.52 \mathrm{~mm}$ diameter using water as the test fluid. Microfin height was $0.20 \mathrm{~mm}$, fin helix angle was $18^{\circ}$, and number of fin starts was 60 . Results were presented for uniform heating conditions in the range 2,000 < Re $<$ 20,000 . Under the same conditions, comparative experiments with an internally smooth tube were also conducted. They noted that the microfin tube provides higher heat transfer performance than the smooth tube although the pressure drop increase is also substantial (in turbulent flow $h_{\text {microfin }} / h_{\text {smooth }}=2.9$ and $\Delta p_{\text {microfin }} / \Delta p_{\text {smooth }}=1.7$ at the maximum Reynolds number tested). The finned tube performance efficiency index as defined by Brognaux et al. [50] were also determined which showed that the heat transfer increase was always superior to the pressure drop penalty. The experimentally obtained Nusselt numbers were empirically correlated separately as a Dittus-Boelter, a SiederTate, and a Gnielinski type correlation. These correlations predicted their data reasonably well.

Wang and Rose [59] compiled an experimental database of twenty-one microfin tubes, covering a wide range of tube and fin geometric dimensions, Reynolds number and including data for water, R11, and ethylene glycol for friction factor for single-phase flow in spirally grooved, horizontal microfin tubes. The tubes had inside diameter at the fin root between $6.46 \mathrm{~mm}$ and $24.13 \mathrm{~mm}$, fin height between $0.13 \mathrm{~mm}$ and $0.47 \mathrm{~mm}$, fin pitch between $0.32 \mathrm{~mm}$ and $1.15 \mathrm{~mm}$, and helix angle between $17^{\circ}$ and $45^{\circ}$. The Reynolds number ranged from $2.0 \times 10^{3}$ to $1.63 \times 10^{5}$. Six earlier friction factor correlations, each based on restricted data sets, were compared with the database as a whole. They reported that none was found to be in good agreement with all of the data and indicated that the Jensen and Vlakancic [57] correlation was found to be the best and represented their database within $\pm 21 \%$.

Han and Lee [60] obtained experimental single-phase heat transfer coefficients and friction factors for four micro finned tubes all with 60 helical fins using liquid water as the test fluid in a double-pipe heat exchanger. The tubes tested had fin helix angles between 9.2 and $25.2^{\circ}$ and fin height between $0.12 \mathrm{~mm}$ and $0.15 \mathrm{~mm}$. Results were presented for cooling conditions in the range $3000<\mathrm{Re}<$ 40,000 and $4<\operatorname{Pr}<7$. Validation experiments with an internally smooth tube were also conducted. Using the heatmomentum transfer analogy, as used by Brognaux et al. [50], they presented their experimentally determined heat transfer and friction factor correlations as functions of the roughness Reynolds number, $\operatorname{Re}_{\varepsilon}$, with a mean deviation and root mean square deviation of less than $6.4 \%$. They noted that the microfin tubes show an earlier achievement of the fully rough region which starts at $\operatorname{Re}_{\varepsilon}=70$ for rough pipes and also validated the finding of Brognaux et al. [50] that the friction factors in microfin tubes do not reach a constant value at high Reynolds numbers as is usually observed in rough pipes surface. No attempt was made to present a direct comparison of heat transfer enhancement between a smooth and a microfinned tube, but an efficiency index was defined. Smaller value of efficiency index means increased friction penalty to establish a given enhancement level. Using this index, the authors noted that the tubes with higher relative roughness and smaller spiral angle show a better heat transfer performance than tubes with larger spiral angle and smaller relative roughness. The authors concluded that the heat transfer area augmentation by higher relative roughness is the main contributor to the efficiency index.

$\mathrm{Li}$ et al. [61] experimentally investigated the single-phase pressure drop and heat transfer in a microfin tube with a $19 \mathrm{~mm}$ outside diameter having 82 helical fins with a fin height of $0.3 \mathrm{~mm}$ and a helix angle of $25.5^{\circ}$ using oil and water as the test fluid in a tube-in-tube heat exchanger. Results were presented for cooling conditions in the range $2500<\operatorname{Re}<90,000$ and $3.2<\operatorname{Pr}<220$. The pressure drop data were collected under adiabatic conditions. Under the same conditions, comparative experiments with an internally smooth tube were also conducted. Their results showed that there is a critical Reynolds number, $\mathrm{Re}_{\mathrm{cr}}$, for heat transfer enhancement. For $\operatorname{Re}<\operatorname{Re}_{\mathrm{cr}}$, the heat transfer in the microfin tube is the same as that in a smooth tube, but for Reynolds numbers higher than $\mathrm{Re}_{\mathrm{cr}}$, the heat transfer in the microfin tube is gradually enhanced compared with a smooth tube. It reaches more than twice that in a smooth tube for Reynolds numbers greater than 30,000 with water as the working fluid. They attributed this behavior to the decrease in the thickness of the viscous sublayer with increasing Reynolds numbers. When the microfins are inside the viscous sublayer, the heat transfer is not enhanced, while when the microfins are higher than the viscous sublayer, heat transfer is enhanced. They also investigated the Prandtl number dependency of the Nusselt number in the form of $\mathrm{Nu} \alpha \mathrm{Pr}^{n}$ and found that the Nusselt number is proportional to $\operatorname{Pr}^{0.56}$ in the enhanced region and is proportional to $\operatorname{Pr}^{0.3}$ in the nonenhanced region. For the high Prandtl number working fluid (oil, $80<\operatorname{Pr}<220$ ), the critical Reynolds number for heat transfer enhancement is about 6000, while for the low Prandtl number working fluid (water, $3.2<\operatorname{Pr}<5.8$ ), the critical Reynolds number for heat transfer enhancement is about 10,000. The reported friction factors in the microfin tube are almost the same as for a smooth tube for Reynolds numbers below 10, 000. They become higher for Re $>10,000$ and reach values $40 \%-50 \%$ greater than that in a smooth tubes for Re $>30,000$. They also concluded that the friction factors in the microfin tube do not behave as in a fully rough tube even at a Reynolds number of 90, 000.

An artificial neural network (ANN) approach was applied by Zdaniuk et al. [62] to correlate experimentally determined Colburn $j$-factors and Fanning friction factors for flow of liquid water in straight tubes with internal helical fins. Experimental data came from eight enhanced tubes reported later in Zdaniuk et al. [63]. The performance of the neural networks was found to be superior compared to 
the corresponding power-law regressions. The ANNs were subsequently used to predict data of other researchers but the results were less accurate. The ANN training database was expanded to include experimental data from two independent investigations. The ANNs trained with the combined database showed satisfactory results, and were superior to algebraic power-law correlations developed with the combined database.

Siddique and Alhazmy [64] also tested a single internally microfinned tube with a nominal inside diameter of $7.38 \mathrm{~mm}$. Microfin height was $0.20 \mathrm{~mm}$, helix angle was $18^{\circ}$, and number of fin starts was 50. Experiments were conducted in a double pipe heat exchanger with water as the cooling as well as the heating fluid for six sets of runs. The pressure drop data were collected under isothermal conditions. Data were taken for turbulent flow with $3300 \leq \mathrm{Re} \leq$ 22,500 and $2.9 \leq \operatorname{Pr} \leq 4.7$. The heat transfer data were correlated by a Dittus-Boelter type correlation, while the pressure drop data were correlated by a Blasius type correlation. These correlations predicted their data to within $9 \%$ and $1 \%$, respectively. The correlation predicted values for both the Nusselt number and the friction factors were compared with other studies. They found that the Nusselt numbers obtained from their correlation fall in the middle region between the Copetti et al. [49] and the Gnielinski [65] smooth tube correlation predicted Nusselt number values. For pressure drop results, they reported the existence of a transition zone for $\operatorname{Re}<11,500$ in which the friction factor data exhibited a local maxima. The presented correlation predicted friction factors values were nearly double that of the Blasius smooth tube correlation predicted friction factors. The authors concluded that the rough tube Gnielinski [65] and Haaland [66] correlations can be used as a good approximation to predict the finned tube Nusselt number and friction factor, respectively, in the tested Reynolds number range.

Zdaniuk et al. [63] experimentally determined the heat transfer coefficients and friction factors for eight helically finned tubes and one smooth tube using liquid water at Reynolds numbers ranging between 12,000 and 60,000. The helically-finned tested tubes had helix angles between $25^{\circ}$ and $48^{\circ}$, number of fin starts between 10 and 45 , and fin height-to-diameter ratios between 0.0199 and 0.0327 . Power-law correlations for Fanning friction and Colburn $j$ factors were developed using a least-squares regression using five simple groups of parameters identified by Webb et al. [58]. The performance of the correlations was evaluated with independent data of Jensen and Vlakancic [57] and Webb et al. [58] with average prediction errors in the $30 \%$ to $40 \%$ range. The authors also gave recommendations about the use of some specific tubes used in their experimentations and concluded that disagreements in the experimental results of Webb et al. [58], Jensen and Vlakancic [57], and their own study imply that a broader database of heat transfer and friction characteristics of flow in helically ribbed tubes is desirable. The authors further recommended that more research should be performed on the influence of geometric parameters on flow patterns, especially in the inter fin region using modern flow visualization techniques or proven computational fluid dynamics (CFD) tools.
In a subsequent analysis, Zdaniuk et al. [67] using genetic programming extended their earlier work [63] presenting a linear regression approach to correlate experimentallydetermined Colburn $j$-factors and Fanning friction factors for flow of liquid water in helically finned tubes. Experimental data came from the eight enhanced tubes used in their previous study [63] discussed above. This new study revealed that, in helically finned tubes, logarithms of both friction and Colburn $j$-factors can be correlated with linear combinations of the same five simple groups of parameters identified in their earlier work [63] and a constant. The proposed functional relationship was tested with independent experimental data yielding excellent results. The authors concluded that the performance of their proposed correlations is much better than that of the power law correlations and only slightly worse than that of the artificial neural networks.

More recently, Webb [68] investigated the heat transfer and friction characteristics of three tubes (19.05 mm O.D., $17.32 \mathrm{~mm}$ I.D.) including one developed by the author (designated as tube T3) having a conical, three-dimensional roughness on the inner tube surface with water flow in the tube. Experiments were conducted in a double tube heat exchanger with water as the cooling as well as the heating fluid. The pressure drop data were collected under adiabatic conditions. The data were taken at a tube side Reynolds number range of 4000-24,000 and the Prandtl number varied from 6.6 to 5.9. The heat transfer data were correlated by a Dittus-Boelter type correlation, while the pressure drop data were correlated by a Blasius type correlation. The measured maximum uncertainty in the friction factor was reported to be $5.96 \%$, while for most data points, the uncertainty in the measured value of the inside heat transfer coefficient was stated to be $8 \%$. The experimentally obtained values for the Nusselt number were compared with an independent study and were found to be $9 \%-12 \%$ higher. The author reported that the TC3 truncated cone tube provides a Nusselt number 3.74 times that of a plain tube, but it has it has nearly $60 \%$ higher pressure drop and concluded that the three-dimensional roughness offers potential for considerably higher heat transfer enhancement (e.g., 50\% higher) than is given by helical ridged tubes. Accelerated particulate fouling data were also provided for TC3 tube, and for five different helical-ribbed tubes for $1300 \mathrm{ppm}$ foulant concentration at $1.07 \mathrm{~m} / \mathrm{s}$ water velocity $(\operatorname{Re}=16,000)$. The fouling rate was compared with helical-rib geometries reported earlier by $\mathrm{Li}$ and Webb [69]. The author noted that the TC3 tube shows a very high accelerated particulate fouling rate, which is higher than that of the helical-ribbed tubes tested by Li and Webb [69] and recommended that the $3-\mathrm{D}$ roughness tubes should experience minimal and acceptable low fouling, if used with relatively clean or treated water.

Most recently, Bharadwaj et al. [70] experimentally determined pressure drop and heat transfer characteristics of liquid water flowing in a single 75 fin start spirally grooved tube (inside diameter $=14.808 \mathrm{~mm}$, fin helix angle $=23^{\circ}$, and fin height $=0.3048 \mathrm{~mm}$ ) with and without a twisted tape insert. Results were presented for uniform heating conditions at $\operatorname{Pr}=5.4$ in the range $300<\operatorname{Re}<35,000$. 
The grooves are clockwise with respect to the direction of flow. The authors noted that for the microfin tube experiments transition-like characteristics begin at $\operatorname{Re} \sim 3000$ and continued up to $\operatorname{Re}=7000$. Beyond this value of Re, the friction factor remained nearly constant similar to flow in a rough tube. Power law-based correlation for the friction factors and Nusselt numbers were presented in the ranges of $300<\operatorname{Re}<3000$ (laminar), $3000<\operatorname{Re}<7000$ (transition), and $\operatorname{Re}>7000$ (turbulent). They noted that in the laminar and turbulent ranges of Re, $\mathrm{Nu}$ is almost doubled compared to its value in a smooth tube as predicted by the Dittus Boelter correlation. But, for the transition range $3000<\mathrm{Re}<$ 7000 , the Nu-data almost coincide with the same smoothtube correlation predicted value, indicating no enhancement in heat transfer in this range. Constant pumping power comparison with smooth tube showed that the spirally grooved tube without twisted tape yields maximum heat transfer enhancement of $400 \%$ in the laminar range and $140 \%$ in the turbulent range. However, for $2500<\mathrm{Re}<9000$, reduction in heat transfer was noticed. For the experiments with the twisted tape inserts having twist ratios of 10.15, 7.95, and 3.4, compared to smooth tube, the heat transfer enhancement due to spiral grooves was found to be even further enhanced. They found that the direction of twist (clockwise and anticlockwise) influences the thermo-hydraulic characteristics. Constant pumping power comparisons with smooth tube characteristics show that in spirally grooved tube with twisted tape, heat transfer increases considerably in laminar and moderately in turbulent range of Reynolds numbers. However, for the spiral tube with anticlockwise twisted tape $(Y=10.15)$, reduction in heat transfer was noticed over the transition range of Reynolds numbers.

From the above comprehensive literature review, it is clear that many studies have been conducted to investigate the heat transfer and pressure drop characteristics of internally finned tubes for single phase situations. However, much of the reported data pertains to large fin systems. Most of the experimental correlations are applicable only for the particular system they were developed for. It is also apparent that quite a few empirical correlations based on experimental data do exist for predicting pressure drop and heat transfer in turbulent flow in finned tubes, but it also seems that there is a substantial disagreement between the results predicted by these different correlations; therefore, a need exists for further research in this area.

\subsubsection{Mathematical Modeling of Fins}

General One-Dimensional Model. Consider a fin having a length $L$ and a cross-sectional area $A_{C}$ extending from a base surface. The thermal conductivity of the fin is $k_{f}$. The convection coefficient for the fin facing the outside fluid stream is $h_{f}$. It is assumed that $h_{f}$ does not vary with position and that the variation of the fin temperature in the transverse direction is negligible. The $x$-axis is directed along the fin center line starting from the base. The heat diffusion equation for the fin is

$$
\frac{1}{A_{c}} \frac{d}{d x}\left(A_{c} \frac{d T_{f}}{d x}\right)-\frac{h_{f}}{k_{f} A_{c}} \frac{d A_{s}}{d x}\left(T_{f}-T_{\infty}\right)=0,
$$

where $T_{f}$ and $T_{\infty}$ are the fin temperature and the external fluid far stream temperature, respectively.

Joint-Fins. Consider a thin wall separating two convective media having free stream temperatures $T_{\infty 1}$ and $T_{\infty 2}$ on the left and right sides, respectively. It is assumed that $T_{\infty 1}>T_{\infty 2}$. The convective media with temperature $T_{\infty 1}$ is refereed to as the "source" while the other one is refereed to as the "sink". Suppose that a very long fin having a uniform cross-sectional area $A_{C}$ penetrates through the wall linking thermally both the source and the sink. The terminology "joint fin" is used to refer to such kinds of fins. It is assumed that the conduction heat transfer at the fin tips on the source and the sink sides are negligible and that the convection coefficient for the fin in the source side is $h_{f 1}$ while it is $h_{f 2}$ on the sink side. Now consider that fin to have a finite length $L$ and insulated from its both ends. The fin heat transfer can be calculated from either the receiver or the sender fin portions. It is equal to the following in a dimensionless form [31]

$$
q_{f}=\frac{\sqrt{h_{f 1} k_{1} P A_{C}}\left(T_{\infty_{1}}-T_{\infty 2}\right) \tanh \left[m_{1} L\left(L_{1} / L\right)\right]}{1+\left(\tanh \left[m_{1} L\left(L_{1} / L\right)\right] / \tanh \left[m_{1} L \sqrt{\left(h_{f 2} / h_{f 1}\right)\left(k_{1} / k_{2}\right)\left(1-\left(L_{1} / L\right)\right)}\right]\right) \sqrt{\left(h_{f 1} / h_{f 2}\right)\left(k_{1} / k_{2}\right)}}
$$

where $m_{1}$ and $m_{2}$ (the receiver and sender fin portion indices) are equal to

$$
m_{1}=\sqrt{\frac{h_{f 1} P}{k_{1} A_{C}}}, \quad m_{2}=\sqrt{\frac{h_{f 2} P}{k_{2} A_{C}}} .
$$

Hairy Fin System. Consider a rectangular fin (primary fin) having a length $L$ and a constant perimeter $P$ and a constant cross-sectional area $A_{C}$. It is extending from a surface which is kept at a base temperature $T_{b}$. A large number of pin rods (secondary fins) are attached on the outer surface of the primary fin. The resulting fin system is referred as to a "hairy fin system". The secondary fins are uniformly distributed over the primary fin surface. The $x$-axis is taken along the length of the primary fin starting from the base cross-section while the $y$-axis is taken in the transverse direction. The one-dimensional conduction heat diffusion equation can be found to be equal to the following [30]:

$$
\frac{d^{2} T_{f}}{d x^{2}}-\left[(1-\phi)+\phi\left(\frac{\pi d}{2}\right) \sqrt{\frac{h_{d} k_{d} d}{h_{f}^{2}}}\right] m^{2}\left(T_{f}-T_{\infty}\right)=0
$$


where $h_{d}, k_{d}, d$, and $T_{f}(x)$ are the convection coefficient between the surface of the secondary fins and the surrounding fluid, thermal conductivity of the secondary fin, secondary fin diameter and the temperature of the main fin at the location of the secondary fin base, respectively. The ratio $\phi$ is equal to the total base area of the secondary fins to the total surface area of the primary fin. The index $m$ is equal to $m=\sqrt{h_{f} P /\left(k_{f} A_{C}\right)}$.

Rooted Fins. Consider a fin having a length $L$ and a uniform cross-sectional area $A_{C}$ extending from the interior surface of a wall having a thickness $L_{1},\left(L_{1}<L\right)$. The temperature of the interior surface is $T_{i}$ while it is equal to $T_{o}$ for the exterior surface. The thermal conductivity of the wall and the fin are $k_{w}$ and $k_{f}$, respectively. The fin portion facing the outside fluid stream is subject to convection with free stream temperature equal to $T_{\infty}$ and convection coefficient of $h_{f}$. It is assumed that $h_{f}$ does not vary with position and that the variation of the fin temperature in the transverse direction is negligible. As such, the performance indicator $\gamma$ can be equal to the following [14]:

$$
\begin{aligned}
& \gamma \\
& =\frac{q_{f}}{\left.\left(q_{f}\right)_{\text {very long }}\right|_{L_{1}=0}}=\frac{-k A_{C} d T /\left.d x\right|_{x=o}}{\sqrt{k_{f} A_{C} h_{f} P\left(T_{o}-T_{\infty}\right)}} \\
& =\frac{1}{m L_{1}}\left\{\frac{1}{\left[\left(\left(T_{i}-T_{\infty}\right) /\left(T_{i}-T_{o}\right)\right)-1\right]}\right. \\
& \left.+\frac{\left[\eta_{o}\left(m L_{1}\right)^{2}\left(L / L_{1}-1\right)-\left[\left(T_{i}-T_{\infty}\right) /\left(T_{i}-T_{o}\right)-1\right]^{-1}\right]}{\left[\sinh \left(n L_{1}\right) / n L_{1}\right]\left[\eta_{o}\left(m L_{1}\right)^{2}\left(L / L_{1}-1\right)+n L_{1} / \tanh \left(n L_{1}\right)\right]}\right\},
\end{aligned}
$$

where $\eta_{o}$ is the efficiency of the fin portion facing the outside stream. The surface with $x=0$ is the fin root base surface. The factor $n$ is equal to

$$
n=\sqrt{\frac{k_{w} S P}{k_{f} A_{C}}}
$$

where $S$ is the shape factor. The unit of $S$ is the reciprocal of the length unit. For example, if the wall temperature reaches its one-dimensional temperature field at an average distance $t$ from the surface of the fin, then $S=1 / t$. The index $m$ is equal to $m=\sqrt{h_{f} P /\left(k_{f} A_{C}\right)}$.

Biconvection Perimeter-Wise Fins. Consider a fin with uniform cross-section $A_{C}$ having a thermal conductivity $k_{f}$. A uniform fin portion of perimeter $P_{1}$ is subject to convection with a free stream temperature of $T_{\infty 1}$ and a convection heat transfer coefficient of $h_{f 1}$ while the remaining fin portion of perimeter $P_{2}$ is subject to another convection medium with $T_{\infty 2}$ and $h_{f 2}$ as the convective parameters. Assuming the temperature variation along the cross-section is negligible, the energy equation can be found to be equal to the following [32]:

$$
\frac{d^{2} T}{d x^{2}}-m^{2}\left(T_{f}-T_{\infty \mathrm{eff}}\right)=0
$$

where

$$
\begin{gathered}
m=\sqrt{\frac{h_{1} P_{1}+h_{2} P_{2}}{k A_{C}}}, \\
T_{\infty \text { eff }}=\frac{T_{\infty 1}+\left(h_{2} P_{2} /\left[h_{1} P_{1}\right]\right) T_{\infty 2}}{1+\left(h_{2} P_{2} /\left[h_{1} P_{1}\right]\right)} .
\end{gathered}
$$

Biconvection Longitudinal-Wise Fins. Consider a fin with uniform cross-section $A_{C}$ and perimeter $P$ having a thermal conductivity $k_{f}$ and a very long length. The fin portion starting from the base and ending a distance $L_{1}$ from the base is subjected to convection with a free stream temperature of $T_{\infty 1}$ and a convection heat transfer coefficient $h_{f 1}$. The remaining portion is subjected to another convective medium with $T_{\infty 2}$ and $h_{f 2}$ as the convective parameters. Assuming the temperature variations along the cross-section is negligible, the fin heat transfer rate can be found to be equal to the following [32]:

$$
\begin{aligned}
q_{f}= & \sqrt{k A_{C} P h_{1}}\left(T_{b}-T_{\infty 1}\right) \\
\times & \left\{\frac{1+\sqrt{h_{1} / h_{2}} \tanh \left(m_{1} L_{1}\right)}{\tanh \left(m_{1} L_{1}\right)+\sqrt{h_{1} / h_{2}}}\right. \\
& \left.\quad-\frac{\left(\left(T_{\infty 1}-T_{\infty 2}\right) /\left(T_{\infty 1}-T_{b}\right)\right) \operatorname{sech}\left(m_{1} L_{1}\right)}{\tanh \left(m_{1} L_{1}\right)+\sqrt{h_{1} / h_{2}}}\right\},
\end{aligned}
$$

where $T_{b}$ is the base temperature, $m_{1}=\sqrt{h_{1} P /\left(k A_{C}\right)}$ and $m_{2}=\sqrt{h_{2} P /\left(k A_{C}\right)}$.

Biconvection Span-Wise Rectangular Fins. Consider a rectangular thin fin having a thermal conductivity $k_{f}$, length $L$ and a thickness $t$. The fin surface is subjected to two different convection conditions. The first region has a span height $H_{1}$ while the second region has a span height $H_{2}$. The convection medium facing the first region has $T_{\infty 1}$ and $h_{f 1}$ as the free stream temperature and the heat transfer convection coefficient, respectively, while $T_{\infty 2}$ and $h_{f 2}$ are those corresponding to the convection conditions for the second region. For very long fin, Khaled [32] showed that the fin total heat transfer is equal to the following:

$$
\begin{aligned}
q_{f}= & m_{2} H_{2} k_{f} t\left(T_{\infty 1}-T_{\infty 2}\right) \\
& -\left(m_{2} H_{2}+m_{1} H_{1}\right) \theta_{1 b} k_{f} t\left(T_{\infty 1}-T_{\infty 2}\right)+k_{f} t\left(T_{\infty 1}-T_{\infty 2}\right) \\
& \times\left\{\frac{\left(\left(h_{2} / h_{1}\right)-1\right) \operatorname{coth}\left(m_{1} H_{1}\right)-\left(\left(h_{1} / h_{2}\right)-1\right) \operatorname{coth}\left(m_{2} H_{2}\right)}{\left(\sqrt{h_{2} / h_{1}} \operatorname{coth}\left(m_{1} H_{1}\right)+\sqrt{h_{1} / h_{2}} \operatorname{coth}\left(m_{2} H_{2}\right)\right)^{2}}\right\},
\end{aligned}
$$

where $m_{1}=\sqrt{h_{1} P_{1} /\left(k A_{C 1}\right)}=\sqrt{h_{1} /(k t)}$ and $m_{2}=$ $\sqrt{h_{2} P_{2} /\left(k A_{C}\right)}=\sqrt{h_{2} /(k t)}, T_{b}$ is fin base temperature and $\theta_{1 b}=\left(T_{b}-T_{\infty 1}\right) /\left(T_{\infty 2}-T_{\infty 1}\right)$. 
Permeable Fins. Consider a permeable fin with uniform cross-section $A_{C}\left(A_{C}=2 H t\right)$ and a perimeter $P(P=2 H)$ having a thermal conductivity $k_{f}$ and a very long length. The fin encounter uniform flow (with density $\rho$ and specific heat $c_{p}$ ) across its thickness with an average suction speed at the upper surface of $\varepsilon V_{o}$ where $\varepsilon$ is the ratio of holes area to the total surface area. The upper surface of the fin is subjected to convection with a free stream temperature of $T_{\infty}$ and a convection coefficient $h_{f}$. The direction of $V_{o}$ is from the upper surface to the lower surface of the fin. Khaled [34] has shown that the fin heat transfer rate is obtained from

$$
\begin{aligned}
\frac{q_{f}}{\left(q_{f}\right)_{\varepsilon=0}} & =\frac{-k_{f}(1-\varepsilon) A_{C} d T_{f} /\left.d x_{f}\right|_{x_{f}=0}}{\sqrt{k_{f} A_{C}\left(h_{f}\right)_{\varepsilon=0} P}\left(T_{B}-T_{\infty}\right)} \\
& =(1-\varepsilon) \sqrt{\left[\frac{\theta^{\prime}(0)}{\left.\theta^{\prime}(0)\right|_{\varepsilon=0}}-\left(\frac{\varepsilon}{1-\varepsilon}\right) \frac{\operatorname{Pr} f_{o}}{\left.\theta^{\prime}(0)\right|_{\varepsilon=0}}\right]},
\end{aligned}
$$

where $\operatorname{Pr}$ is the external fluid Prandtl number. He also derived a correlation for $\theta^{\prime}(0)$ using similarity solution for the boundary layer problem above the upper surface of the fin. This correlation is equal to

$$
\begin{gathered}
-\theta^{\prime}(0)=\frac{b_{1} \operatorname{Pr}^{b_{2}}\left(\operatorname{Pr}^{b_{3}}+b_{4}\left(\varepsilon f_{o}\right)^{b_{5}}\right)^{b_{6}}}{\left(1+b_{7} \operatorname{Pr}^{b_{8}}\right)} \\
b_{1}=1.0605 \quad b_{2}=1.1424 \quad b_{3}=-1.6070 \\
b_{4}=4.7261 \quad b_{5}=2.3293 \quad b_{6}=0.3782 \\
b_{7}=0.6104 \quad b_{8}=0.4466 \\
\operatorname{Re}_{H}=\frac{\rho u_{\infty 0} H}{\mu} \quad f_{o}=\left(\frac{V_{o}}{u_{\infty o}}\right) \sqrt{\operatorname{Re}_{H}} .
\end{gathered}
$$

The velocities $u_{\infty o}$ and $V_{o}$ are equal to the following:

$$
\begin{gathered}
u_{\infty o}=\frac{V H}{l}\left(1-\frac{\varepsilon}{24}\left(\frac{d}{t}\right)\left[\frac{\rho V d}{\mu}\right]\right), \\
V_{o}=\frac{1}{24}\left(\frac{d}{t}\right)\left(\frac{\rho V d}{\mu}\right) V,
\end{gathered}
$$

where $d$ and $V$ are the far stream external normal velocity and $d$ is the diameter of the holes on the fin, respectively. $\mu$ is the dynamic viscosity of the external fluid.

Porous Fins. Heat transfer in porous fins has also gained a recent attention of many researchers. Porous materials of high thermal conductivity have been used to enhance heat transfer as will be discussed in Section 3.2. Kiwan and Al-Nimr [33] were among the recent researchers who numerically investigated the effect of using porous fins on the heat transfer from a heated horizontal surface. The basic philosophy behind this kind of enhancers is to increase the effective area through which heat is convected to ambient fluid in addition to augment the convection heat transfer coefficient by increasing mixing or the thermal dispersions levels between the ambient fluid and the solid phase of the porous fin. They found that using a porous fin with certain porosity might give same performance as conventional fin and save 100 times porosity percent of the fin material. In addition, Kiwan and Zeitoun [71] found that porous fins enhanced the heat transfer coefficient more than $70 \%$ compared to that of conventional solid fins under natural convection conditions.

Capsulated Liquid Metal Fins. A capsulated fin is a capsule full of a liquid metal made of a very thin metal shell of very high thermal conductivity. The capsulated fins are attached to hot surfaces in different manners and directions, which allow for the activation of natural convective currents in the liquid metal held inside the capsules. There must be some temperature increase in the direction of the gravitational force to activate the free convective currents within the liquid metal. Aldoss et al. [72] introduced this novel type of heat transfer enhancement technique for the first time, and numerically estimated and compared the thermal performance of a liquid metal capsulated fin with that of a conventional solid fin, investigating the effect of several design and operating parameters. Two equal-size geometries for the capsulated fins longitudinal sectional area were considered: the rectangular and the halfcircular fins. It was found that using capsulated fins might enhance the performance over an equal-size conventional solid fin by about $500 \%$ for the conventional steel fin, $270 \%$ for the conventional solid sodium fin, and $150 \%$ for the conventional aluminum fin for a fin length to width ratio of 5. Using capsulated fins is justified in applications that involve a high-base temperature, high height-to-width aspect ratio, and a high external convective heat transfer coefficient.

Models for Heat Transfer Correlations for Microfins. In experimental work on turbulent single-phase heat transfer in internally finned tubes, most of the heat transfer correlations can be broadly classified as simple nondimensional empirical correlations, compounded/modified nondimensional empirical correlations containing additional variables adjudged to be of controlling importance by the researcher, and lastly correlations based on the heat-momentum transfer analogy model for rough surfaces as first proposed by Dipprey and Sabersky [73] are used for microfinned tubes.

The simple nondimensional correlations use the governing differential conservation equations of the tube side fluid turbulent boundary layer as a basis for defining the appropriate nondimensional groups for correlating the heat transfer data. Thus, the Nusselt number is proposed as a function of the Reynolds and Prandtl numbers. The heat transfer data from these investigations are typically correlated by a relationship of the form

$$
\mathrm{Nu}=\frac{h d}{k}=C \operatorname{Re}^{m} \operatorname{Pr}^{n},
$$

the so-called Dittus Boelter type correlation. The variables $h, d$ and $k$ are the fully developed convection heat transfer 
coefficient, pipe inner diameter, and the fluid thermal conductivity, respectively. The experimental data is then used to find coefficients $C, m$, and $n$ using regression analysis or cross-plotting.

In experimental works where very limited testing has been done, for example, using only a single microfinned tube, very simple correlations of the Dittus Boelter type have been proposed to explain the data trend. A good example of this type of a correlation is found in the work of Chiou et al. [55], in which the average Nusselt numbers from the experimentally obtained data were correlated as

$$
\mathrm{Nu}=0.043 \operatorname{Re}^{0.8} \operatorname{Pr}^{0.4}
$$

In the same way Copetti et al. [49], have correlated their heat transfer data as

$$
\mathrm{Nu}=0.0034 \operatorname{Re}^{1.1} \operatorname{Pr}^{0.4}
$$

Another variation of this approach is seen in the work of AlFahed et al. [54] who correlated the average Nusselt numbers from their experimentally obtained data in a Sider-Tate type correlation as

$$
\mathrm{Nu}=0.039 \operatorname{Re}^{0.8} \operatorname{Pr}^{1 / 3}\left(\frac{\mu_{b}}{\mu_{w}}\right)^{0.14} .
$$

As stated above, the compounded/modified nondimensional empirical correlations contain additional variables adjudged to be of controlling importance by the researcher. A good example of this approach is seen in the work of Carnavos [45], who conducted an extensive experimental investigation using eleven internally finned tubes with air, water, and ethylene glycol-water solutions. His heat transfer correlation is given as

$$
\mathrm{Nu}=0.023 \operatorname{Re}^{0.8} \operatorname{Pr}^{0.4} F,
$$

where

$$
F=\left(\frac{A_{\mathrm{fa}}}{A_{\mathrm{fc}}}\right)^{0.1}\left(\frac{A_{n}}{A_{a}}\right)^{0.5}[\sec (\alpha)]^{2} .
$$

We see that this heat transfer correlation is in the form of a modified Dittus-Boelter single-phase correlation having an additional correction factor " $F$ ", consisting of the ratios of the actual flow area $\left(A_{\mathrm{fa}}\right)$ to the core flow area $\left(A_{\mathrm{fc}}\right)$ and the nominal heat transfer area $\left(A_{n}\right)$ to-the actual heat transfer area $\left(A_{a}\right)$, respectively. The secant of the helix angle $(\alpha)$ raised to the third power is also included in " $F$ ".

Another variation of nondimensional correlation type is to fit the heat transfer data in terms of the so called Colburn j-factor, modified by additional variables adjudged to be of controlling importance. An example of this can be seen in the work of Zdaniuk et al. [63] who experimentally determined the heat transfer coefficients and friction factors for eight helically-finned tubes and one smooth tube using liquid water. Their heat transfer correlation is given as

$$
j=\operatorname{St} \operatorname{Pr}^{2 / 3}=0.029 \operatorname{Re}^{-0.347} N_{s}^{0.252}\left(\frac{e}{D}\right)^{0.0877} \alpha^{0.362},
$$

where $N_{s}$ is the number of fin starts, $e / D$ is the fin height to diameter ratio, and $\alpha$ is the helix angle.

Using the heat-momentum transfer analogy as applied for smooth surfaces, Dipprey and Sabersky [73] showed that a similar analogy model is applicable for sand-grain type wall roughness. For turbulent flow in a rough circular tube, this can be given as

$$
\mathrm{Nu}=\frac{(f / 2) \operatorname{Re} \operatorname{Pr}}{1+(f / 2)^{0.5}\left[g\left(e^{+}\right) \operatorname{Pr}^{n}-B\left(e^{+}\right)\right]},
$$

where the Nusselt number is a function of Reynolds and Prandtl numbers and the friction factor $(f)$ which depends on the geometrical variables $\left(e / D, N_{s}\right.$ and $\alpha$ ) of the microfin tube. $B\left(e^{+}\right)$is the friction similarity function for the rough/microfinned tube where $e^{+}$is the roughness Reynolds number, while the $g\left(e^{+}\right)$is the correlating function for the rough/microfinned tube determined separately. These functions will be different for different roughness types, that is, for geometrically different microfinned tubes.

An example of this type of heat transfer correlation is found in the work of Copetti et al. [49], who have also correlated their microfinned tube heat transfer data as

$$
\mathrm{Nu}=\frac{(f / 2)(\mathrm{Re}-1000) \operatorname{Pr}}{1+(f / 2)^{0.5}\left[8.05 \operatorname{Pr}^{-0.38}+9.09\right]} .
$$

\subsection{Heat Transfer Enhancement Using Porous Media}

3.2.1. Introduction. Porous media are used to enhance the rate of heat and mass transfer in energy systems. The values of Nusselt number in presence of porous media are approximately 50\% above the values predicted for laminar flows in channels without porous materials [74]. Moreover, the convective heat transfer coefficient is larger for systems filled with porous material than the systems without porous material due to the large thermal conductivity of the porous matrix compared with the fluid thermal conductivity, especially for gas flows. However, porous media results in substantial pressure drop [9]. To minimize pressure drop, partially fillings of porous media can be used.

Partial filling of the porous medium has advantage of reducing the pressure drop compared with a system filled completely with porous medium [9]. A partial filling of a channel with porous media redirects the flow to escape from the core porous region, depending on the permeability of the porous medium, to the outer region. This effect reduces the boundary layer thickness. As such, the rate of heat transfer is enhanced. Moreover, the porous medium increases the effective thermal conductivity and heat capacity of the fluid-porous material medium and the solid matrix enhances the rate of radiative heat transfer in a system especially if the gas is the working fluid. In summary, the heat transfer enhancements associated with partial filing of the porous medium take place by three mechanisms: (i) flow redistribution, (ii) thermal conductivity modification, and (iii) radiative property modification of the medium.

Many works have been conducted in the domain of partially fillings of porous media. For example, Jang and 
Chen [75] conducted a numerical study for a forced flow in a parallel channel partially filled with a porous medium by adopting the Darcy-Brinkman-Forchheimer model with a thermal dispersion term. Chikh et al. [76, 77] presented an analytical solution for the fully developed flow in annulus configuration partially filled with porous medium. Al-Nimr and Alkam [78] extended the analysis to the transient solution for annulus flow with porous layer. They found that an increase of up to 12 times in the Nu number was reported when annuli partially filled with porous substrates located either on the inner or the outer cylinder in comparison with the clear annuli. Alkam and Al-Nimr [79] further investigated the thermal performance of a conventional concentric tube heat exchanger by placing porous substrates on both sides of the inner cylinder. Numerical results obtained showed that porous substrates of optimum thicknesses yield the maximum improvement in the heat exchanger performance with moderate increase in the pumping power. This kind of heat transfer enhancers are used in wide range of practical applications including: (a) forced channel flow applications [80-84] and (b) renewable energy applications $[82,85]$. Recent reviews of the subject are available in $[74,86]$.

\subsubsection{Mathematical Modelings}

Darcy Law. Darcy law is one of the earliest flow transport models in porous media. In his experiments on steadystate unidirectional flow in a uniform medium, Darcy [87] revealed proportionality between flow rate and the applied pressure difference. In modern notation, Darcy is expressed by

$$
u=-\frac{K}{\mu} \frac{\partial P}{\partial x}
$$

where $u, P, \mu$ and $K$ are the Darcy velocity, fluid pressure, dynamic viscosity of the fluid and the permeability of the porous medium, respectively.

Brinkman's Equation. As seen from (24), Darcy law ignores the boundary effects on the flow. This assumption may not be valid especially when the boundaries of the porous medium are close to each other. Therefore, the following is used as an extension for the Darcy equation for unidirectional flow

$$
\nabla P=-\frac{\mu}{K} \mathbf{v}+\tilde{\mu} \nabla^{2} \mathbf{v} .
$$

Equation (25) has been referred in the literature [88, 89] as "Brinkman equation". The first viscous term on the right is the Darcy term while the second term in the right is analogous to the momentum diffusion term in the Navier Stokes equation with $\tilde{\mu}$ as the effective dynamic viscosity of the medium. For isotropic porous medium, Bear and Bachmat [90] showed that the effective viscosity is related to the porosity through the following relation:

$$
\frac{\tilde{\mu}}{\mu}=\frac{1}{\varepsilon T^{*}},
$$

where $\varepsilon$ and $T^{*}$ are the porosity (void-volume-to-thetotal-medium-volume ratio) and tortuosity of the medium, respectively.

Generalized Flow Transport Model. In cases where fluid inertia is not negligible, another drag force starts to become significant which is the form drag excreted by the fluid on the solid. Vafai and Tien [91] suggested a generalized model for flow transport in porous media based on Brinkman and Forchheimer's equations. The latter equation takes into considerations the presence of form drags due fluid inertia. This generalized model is summarized in the following equation:

$$
\begin{aligned}
\rho_{f}\left[\frac{1}{\varepsilon} \frac{\partial \mathbf{v}}{\partial t}+\frac{1}{\varepsilon} \nabla \cdot\left(\frac{\mathbf{v}}{\varepsilon}\right)\right]= & -\frac{1}{\varepsilon} \nabla(\varepsilon P)+\frac{\mu}{\varepsilon \rho_{f}} \nabla^{2} \mathbf{v} \\
& -\frac{\mu}{K} \mathbf{v}-\frac{c_{F} \rho_{f}}{K^{1 / 2}}|\mathbf{v}| \mathbf{v},
\end{aligned}
$$

where $c_{F}$ and $\rho_{f}$ are the dimensionless form-drag constant and the fluid density, respectively. For packed bed of solid spheres of diameter $d_{p}, K$ and $c_{F}$ are equal to the following:

$$
K=\frac{\mathcal{\varepsilon}^{3} \cdot d_{p}^{2}}{150(1-\varepsilon)^{2}} ; \quad c_{F}=\frac{1.75}{\sqrt{150 \varepsilon^{3}}} .
$$

In addition, the previous equation did not neglect flow convective terms as does (24) and (25), the terms on the left side. Note that the last term on the right represents the form drag term. The previous equation is usually referred as Brinkman-Forcheiner's equation (Table 1).

Heat Transfer inside Porous Media. Following the works of Amiri and Vafai [8, 92] and Alazmi and Vafai [93] and based on the principle of local thermal nonequilibrium conditions between the fluid and the solid, the energy equations for both the solid and fluid are equal to

$$
\begin{aligned}
& \varepsilon\left(\rho c_{p}\right)_{f}\left(\frac{\langle T\rangle^{f}}{\partial \tau}+\mathbf{u}_{f} \cdot \nabla\langle T\rangle^{f}\right) \\
& =\nabla \cdot\left(\mathbf{k}_{f}^{a} \cdot \nabla\langle T\rangle^{f}\right)+h_{f s}\left(\langle T\rangle^{s}-\langle T\rangle^{f}\right), \\
& (1-\varepsilon)\left(\rho c_{p}\right)_{s} \frac{\langle T\rangle^{s}}{\partial \tau}=\nabla \cdot\left(\mathbf{k}_{s}^{a} \cdot \nabla\langle T\rangle^{s}\right)+h_{f s}\left(\langle T\rangle^{f}-\langle T\rangle^{s}\right),
\end{aligned}
$$

where $\langle T\rangle^{f},\langle T\rangle^{s}, \mathbf{u}_{f}, \mathbf{k}_{f}^{a}, \mathbf{k}_{s}^{a}, \varepsilon$, and $h_{f s}$ are the local fluid averaged temperature, local solid averaged temperature, fluid velocity vector, fluid effective thermal conductivity tensor, solid effective thermal conductivity tensor, porosity of the tensor and interstitial convective heat transfer coefficient. As seen from (29), the energy equations for both phases are coupled by the interstitial convective heat transfer between the fluid and the solid. The concept of local thermal nonequilibrium and fluid thermal dispersion are well established in the theory of porous media. Examples on corresponding researches can be found in the works of Amiri and Vafai $[8,92]$ and Alazmi and Vafai [93]. 
TABLE 1: Summary of the flow transport models in porous media [94].

\begin{tabular}{lcc}
\hline Flow transport model & Equation & Features \\
\hline Darcy's law & $(24)$ & Simple; Consider Darcy resistance; Neglect B.Cs.; Neglect form drag; Neglect convections \\
Brinkman's equation & $(25)$ & Consider Darcy resistance; Consider B.Cs.; Neglect form drag; Neglect convections \\
Brinkman-Forcheimer's equation & $(27)$ & Consider Darcy resistance; Consider B.Cs.; consider form drag; consider convections \\
\hline
\end{tabular}

B.Cs.: Boundary conditions effects.

In applications involving porous media of small sizes of both the pores and the solid particles and as illustrated in $[8,92]$, Khanafer and Vafai [95] and Marafie and Vafai [96], local thermal equilibrium may serve as a good approximation for the temperature field. In these applications, the solid temperatures as well as fluid temperatures are the same and (29) reduces to the following equation:

$$
\begin{gathered}
{\left[\rho_{s} c_{p}(1-\varepsilon)+\rho_{f} c_{p f} \varepsilon\right] \frac{\partial T}{\partial t}+\varepsilon\left(\rho c_{p}\right)_{f} \mathbf{u}_{f} \cdot \nabla T} \\
=\nabla\left(\left[\mathbf{k}_{s}^{a}+\mathbf{k}_{f}^{a}\right] \cdot \nabla T\right) .
\end{gathered}
$$

The second term on the left is responsible for heat transfer due to convection. The thermal conductivity tensors for isotropic materials are equal to the following:

$$
\mathbf{k}_{s}^{a}=(1-\varepsilon) k_{s} ; \quad \mathbf{k}_{b}^{a}=\left(\varepsilon+0.5 \operatorname{Pr}\left[\frac{\rho_{f} u_{f} d}{\mu_{f}}\right]\right) k_{b} .
$$

3.3. Heat Transfer Enhancement Using Fluids with Large Particles Suspensions. Huge number of investigations has been carried out in the past in order to seek for developing novel passive methods for enhancing the effective thermal conductivity of the fluid or increasing the convection heat transfer coefficient. One of the methods is introducing into the base liquid high thermally conductive particulate solids such as metals or metal oxides. Examples of these investigations according to Ding et al. [10] are seen in the works of Sohn and Chen [97], Ahuja [98, 99] and Hetsroni and Rozenblit [100]. These early investigations used suspensions of millimeter or micrometer sized particles. They showed some enhancement. However, they introduced problems to the thermal system such abrasion and channel clogging due to poor suspension stability especially in the case of mini- and/or microchannels. A new passive method developed by Choi [101] which is termed "nanofluids" has shown to resolve some disadvantages associated with the suspensions of large particles.

\subsection{Heat Transfer Enhancement Using Nanofluids}

3.4.1. Introduction. Nanofluids are fluids that contain suspensions of nanoparticles of high thermally conductive materials like carbon, metals, and metal oxides into heat transfer fluids to improve the overall thermal conductivity. These nanoparticles are usually of order $100 \mathrm{~nm}$ or less. Nanoparticles could be either spherical or cylindrical like carbon multiwalled nanotubes [102]. The advantages of properly engineered nanofluids according to Ding et al. [10] include the following: (a) higher thermal conductivities than that predicted by currently available macroscopic models,

(b) excellent stability,

(c) little penalty due to an increase in pressure drop, and

(d) little penalty due to an increase in pipe wall abrasion experienced by suspensions of millimeter or micrometer particles.

The suspensions of nanoparticles in nanofluids are found to increase the effective thermal conductivity of the fluid under macroscopically static conditions. Numerous studies have been carried on this aspect [101, 103-111]. On the other hand, there are few number of studies have been conducted on other aspects like phase change behaviour of nanofluids [112-117]. Moreover, there are few researches that demonstrate the enhancement in the convection heat transfer coefficient caused by nanofluids [118-124].

The enhancements in thermal conductivity of nanofluids are due to the fact that particles surface area to volume ratio increases as the diameter decreases. This effect tends to increase the overall exposed heat transfer surface area for a given concentration of particles as their diameters decreases. Further, the presence of nanoparticles suspensions in fluids tend to increase the mixing effects within the fluid which produce additional increase in the fluid's thermal conductivity due to thermal dispersion effects as discussed by Xuan and Li [122].

Nanofluids possess a large effective thermal conductivity for very low nanoparticles concentrations. For instance, the effective thermal conductivity of ethylene glycol is increased by up to $40 \%$ percent higher than that of the base fluid when a 0.3 volumetric percent of copper nanoparticles of mean diameter less than $10 \mathrm{~nm}$ are suspended in it [125]. This enhancement is expected to be more as the flow speed increases resulting in an increase in the thermal dispersion effect [120]. Lee et al. [105] measured the effective thermal conductivity of $\mathrm{Al}_{2} \mathrm{O}_{3}$ and $\mathrm{CuO}$ suspended nanoparticles in water and ethylene glycol. They found out that the effective thermal conductivity was enhanced by more than $20 \%$ when a $4 \%$ volume of $\mathrm{CuO} /$ ethylene glycol mixture was used.

Ding et al. [10] indicated that Xuan and Li [122] showed that the convection heat transfer coefficient was increased by $\sim 60 \%$ for an aqueous-based nanofluid of $2 \%$ $\mathrm{Cu}$ nanoparticles by volume, but the nanofluid only had an effective thermal conductivity approximately 12.5\% higher than that of the base liquid. Also, they indicated that Wen and Ding [124] observed a $\sim 47 \%$ increase in the convective heat transfer coefficient of aqueous c-alumina nanofluids at $x / D \sim 60$ for 1.6 vol.\% nanoparticle loading and $\operatorname{Re}=1600$, 
which was much greater than that due to the enhancement of thermal conduction $(<\sim 10 \%)$. Amazingly, Ding et al. [10] showed that nanofluids containing 0.5 wt.\% of carbon nanotubes (CNT) produced enhancement in convection heat transfer which may be over $350 \%$ of that of the base liquid at $\operatorname{Re}=800$, and the maximum enhancement occurs at an axial distance of approximately 110 times the tube diameter. This increase is much greater than that due to the enhancement of thermal conduction $(<\sim 40 \%)$. The observed large enhancement of the convective heat transfer coefficient is associated with the following reasons:

(a) enhancement of the thermal conductivity under the static conditions,

(b) further enhancement on the thermal conduction under the dynamic conditions (shear induced),

(c) reduction of the boundary layer thickness and delay in the boundary layer development,

(d) particle re-arrangement due to non-uniform shearrate across the pipe cross-section, and

(e) high aspect ratio of carbon nanotubes.

The thermal capacity of nanofluids $\left(\rho C_{p}\right)_{\mathrm{nf}}$ is equal to

$$
\left(\rho C_{p}\right)_{\mathrm{nf}}=(1-\phi)\left(\rho C_{p}\right)_{\mathrm{bf}}+\phi\left(\rho C_{p}\right)_{p},
$$

where the subscript nf, bf and $p$ denote the nanofluid or the dispersive region, base fluid and the particles, respectively. The parameter $\phi$ is the nanoparticles volume fraction which represents the ratio of the nanoparticles volume to the total volume of the nanofluid. A nanofluid composed of pure water and copper nanoparticles suspensions with $2 \%$ volume fraction has a value of $\left(\rho C_{p}\right)_{\text {nf }}$ equal to $99 \%$ that for the pure water which is almost the same as the thermal capacity of the pure fluid.

3.4.2. Effective Thermal Conductivity of Nanofluids. One of the elementary models of the effective thermal conductivity of nanofluids is that of Xuan and Roetzel [120]. They suggested the following mathematical model for the effective thermal conductivity of the nanofluid, $k_{\mathrm{nf}}$

$$
k_{\mathrm{nf}}=\left(k_{\mathrm{nf}}\right)_{o}+C^{*}\left(\rho c_{p}\right)_{\mathrm{nf}} \phi h u,
$$

where $C^{*}$ is a constant depending on the diameter of the nanoparticle and its surface geometry. The constant $\left(k_{\mathrm{nf}}\right)_{o}$ represents the effective thermal conductivity of the nanofluid under stagnant conditions where the bulk velocity $u$ is equal to zero. This constant is proposed by Xuan and Roetzel [120] to be equal to that predicted from the Maxwell model for effective thermal conductivity of solid-liquid mixtures for micro or millimeter sized particles suspended in base fluids [126]. It has the following form:

$$
\frac{\left(k_{\mathrm{nf}}\right)_{o}}{k_{\mathrm{bf}}}=\frac{k_{p}+2 k_{\mathrm{bf}}-2 \phi\left(k_{\mathrm{bf}}-k_{p}\right)}{k_{p}+2 k_{\mathrm{bf}}+\phi\left(k_{\mathrm{bf}}-k_{p}\right)},
$$

where $k_{p}$ and $k_{\mathrm{bf}}$ are the thermal conductivity of the nanoparticles and the base fluid, respectively. According to formula (34), 2.0\% volume fraction of copper particles produces $8.0 \%$ increase in $\left(k_{\mathrm{nf}}\right)_{o}$ when compared to the thermal conductivity of the pure fluid. However, Das et al. [114] demonstrated that the $1 \%$ particle volumetric concentration of $\mathrm{CuO} /$ water nanofluids, the thermal conductivity ratio increased from $6.5 \%$ to $29 \%$ over a temperature range of $21-51^{\circ} \mathrm{C}$. Liu et al. [127] synthesized a copper nanofluid which showed thermal conductivity enhancement of $23.8 \%$ for $0.1 \%$ volumetric concentration of copper particles in water. Therefore, new studies have been implemented for seeking new models for the effective thermal conductivity of nanofluids.

Of these studies is the study of Yu and Choi [128]. They proposed a renovated Maxwell model which includes the effect of the effect of the nanolayer surrounding nanoparticles. They found that this nanolayer has a major role on the effective thermal conductivity of nanofluids when the particle diameter of less than $10 \mathrm{~nm}$. The effects of the surface adsorption of nanoparticles on the thermal conductivity of the nanofluid were modelled by Wang et al. [110]. They showed a good agreement between the model and their experiment for $50 \mathrm{~nm} \mathrm{CuO} /$ deionized water of dilute concentration $(<0.5 \%)$. Koo and Kleinstreuer [129, 130] presented a thermal conductivity model for nanofluids comprising a static part and a dynamic part due to the Brownian motion of nanoparticles. The thermal conductivity model of nanofluids developed by Hamilton and Crosser [131] and the Bruggemen [132] model was found to be different by the thermal conductivity measurement data of Murshed et al. [133] by about $17 \%$ for a $5 \%$ particle volumetric concentration.

Researches have also took in their account the role of the effective thermal conductivity of the interfacial shell between the nanoparticle and the base fluid as can be seen in the work of Xue and $\mathrm{Xu}$ [134]. The effective thermal conductivity model of nanofluids proposed by Chon et al. [135] was developed as a function of Prandtl number, particle Reynolds number based on the Brownian velocity, thermal conductivity of the particle and base fluid, volume fraction and particle size. Moreover, Prasher et al. [136] presented a Brownian motion based convective-conductive model for the effective thermal conductivity of nanofluids. The nanofluid thermal conductivity developed model of Jang and Choi [137] took into account the collision between base fluid molecules, thermal diffusion of nanoparticles in fluids, collision between nanoparticles and nanoconvection due to Brownian motion. A comprehensive review of experimental and theoretical investigations on the thermal conductivity of nanofluids by various researchers was compiled by Wang and Mujumdar [138] and Vajjha and Das [139]. The previously developed models are presented in the works of Vajjha and Das [139].

It should be mentioned that Buongiorno [140] considered seven slip mechanisms that can produce a relative velocity between the nanoparticles and the base fluid: inertia, Brownian diffusion, thermophoresis, diffusiophoresis, Magnus effect, fluid drainage, and gravity. Of all of these 
mechanisms, only Brownian diffusion and thermophoresis were found to be important. Buongiorno's [140] analysis consisted of a two-component equilibrium model for mass, momentum, and heat transport in nanofluids. He found that a nondimensional analysis of the equations implied that energy transfer by nanoparticle dispersion is negligible and cannot explain the abnormal heat transfer coefficient increases. That is the second term of (33) on the right side is negligible. Buongiorno suggests that the boundary layer has different properties because of the effect of temperature and thermophoresis. The viscosity may be decreasing in the boundary layer, which would lead to heat transfer enhancement according to the analysis of Buongiorno [140]. Although Buongiorno [140] found out that thermal dispersion mechanism for enhancing heat transfer in convective conditions is negligible, many researches still adopt the fact that it is a major mechanism. Example of these works is the recent work of Mokmeli and Saffar-Avval [141].

Recently, Vajjha and Das [139] developed a model for thermal conductivity of three nanofluids containing aluminum oxide, copper oxide, and zinc oxide nanoparticles dispersed in a base fluid of 60:40 (by mass) ethylene glycol and water mixture. The developed is a refinement of an existing model, which incorporates the classical Maxwell model and the Brownian motion effect to account for the thermal conductivity of nanofluids as a function of temperature, particle volumetric concentration, the properties of nanoparticles, and the base fluid. The developed model agrees well with the experimental data. The several existing models for thermal conductivity were compared with the experimental data obtained from these nanofluids, and they do not exhibit good agreement except for the model developed by Koo and Kleinstreuer [129].

Vajiha and Das [139] Effective Thermal Conductivity Model for Nanofluids. The model of Vajjha and Das for the thermal conductivity model of nanofluids is presented in the following equation:

$$
\begin{aligned}
\left(k_{\mathrm{nf}}\right)_{o}= & \frac{k_{p}+2 k_{\mathrm{bf}}-2 \phi\left(k_{\mathrm{bf}}-k_{p}\right)}{k_{p}+2 k_{\mathrm{bf}}+2 \phi\left(k_{\mathrm{bf}}-k_{p}\right)} k_{\mathrm{bf}} \\
& +\left(5 \times 10^{4}\right) \beta \phi\left(\rho_{\mathrm{bf}}\right)\left(C_{p}\right)_{\mathrm{bf}} \sqrt{\frac{\kappa T}{\rho_{p} d_{p}}} f(T, \phi), \\
f(T, \phi)= & \left(2.8127 \times 10^{-2} \phi+3.917 \times 10^{-2}\right)\left(\frac{T}{T_{o}}\right) \\
& +\left(-3.0669 \times 10^{-2} \phi-3.91123 \times 10^{-3}\right),
\end{aligned}
$$

where $\kappa$ is the Boltzmann constant $\left(\kappa=1.381 \times 10^{-23} \mathrm{~J} / \mathrm{K}\right)$, $T_{o}$ is a reference temperature $\left(T_{o}=273 \mathrm{~K}\right),\left(C_{p}\right)_{\mathrm{bf}}$ is the specific heat of the base fluid, $\rho_{\mathrm{bf}}$ is the base fluid density, $\rho_{p}$ is the density of the nanoparticle and $d_{p}$ is the nanoparticle diameter. Note that the base fluid is 60:40-by massethylene glycol and water mixture (Table 2).

3.4.3. Summary of Literature on Nanofluids. All of the research on heat transfer in nanofluids reported increases in heat transfer due to the addition of nanoparticles in the base fluid. To what degree and by what mechanism is still debatable. However, the following trends were in general agreement with all researchers [142].

(i) There is an enhancement in the heat transfer coefficient with increasing Reynolds number.

(ii) The heat transfer coefficient enhancement increases with decreasing nanoparticle size.

(iii) The heat transfer coefficient enhancement increases with increasing fluid temperature (more than just the base fluid alone).

(iv) The heat transfer coefficient enhancement increases with increasing nanoparticle volume fraction.

Some nanofluid researches conflict. Below are some explanations as to why there might be such a discrepancy between results [142].

A. Aggregation. It has been shown that nanoparticles tend to aggregate quite quickly in nanofluids, which can impact the thermal conductivity and the viscosity of the nanofluid. Not all researchers account for this whether it is through experimental or numerical research.

B. Unknown Nanoparticle Size Distribution. Researchers rarely report the size distribution of nanoparticles or aggregates-they only list one nanoparticle size-which could affect results. Many researchers do not measure the nanoparticles themselves, and rely on the manufacturer to report this information.

C. Differences in Theory. Researchers have not agreed upon which heat transfer mechanisms are important, dominate, and how they should be accounted for in calculations. The discrepancy leads to different analyses and different results.

D. Different Nanofluid Preparation Techniques. Depending on how the nanofluids are made, for instance whether it is by a one-step of two-step method, the dispersion of the nanofluids could be effected. Some researchers coat the nanoparticles to inhibit agglomeration, while others do not.

3.5. Heat Transfer Enhancement Using Phase-Change Devices. A heat pipe is an efficient compact device with a simple structure and no moving parts that allows the transfer of a large amount of heat from various engineering systems through a small surface area. It basically consists of a duct closed at both ends whose inside wall is covered with a layer of a porous wicking material saturated with the liquid phase of the working fluid while the vapor phase fills the central core of the duct. Heat is transferred from one end (the evaporator) of the pipe to the other (the condenser) by evaporation from the wick at the evaporator, flow of vapor through the core to the condenser, condensation on the wick in the condenser, and return flow of liquid by capillary action in the wick back to the evaporator. If the 
Table 2: $\beta$-Functions and Variable Ranges for Three Nanofluids, 60 : 40 (by Mass) Ethylene Glycol and Water Mixture [139].

\begin{tabular}{lccc}
\hline Type of particles & $\beta$ & Concentration & Temperature \\
\hline $\mathrm{Al}_{2} \mathrm{O}_{3}$ & $8.4407(100 \phi)^{-1.07304}$ & $1 \% \leq \phi \leq 10 \%$ & $298 \mathrm{~K} \leq T \leq 2363 \mathrm{~K}$ \\
$\mathrm{ZnO}$ & $8.4407(100 \phi)^{-1.07304}$ & $1 \% \leq \phi \leq 7 \%$ & $298 \mathrm{~K} \leq T \leq 2363 \mathrm{~K}$ \\
$\mathrm{CuO}$ & $9.881(100 \phi)^{-0.9446}$ & $1 \% \leq \phi \leq 6 \%$ & $298 \mathrm{~K} \leq T \leq 2363 \mathrm{~K}$ \\
\hline
\end{tabular}

condenser is above the evaporator, then the liquid returns under gravity to the evaporator and the need for a wick can be avoided. A heat source and a heat sink usually differing by a small temperature difference are present at the ends of the heat pipe. The heat pipe performance depends on the flow rates of the vapor and liquid, generally requiring the pressure gradient in the vapor to be negative, and positive in the liquid as provided by the self-pumping ability of the wick material. The heat transfer capability of a heat pipe is mainly related to the transport properties of selected working fluid, system operating pressure, and wick porosity characteristics. Since the earliest theoretical analysis of heat pipes was presented by Cotter [143], a lot of work of research has been conducted on heat pipes. Vasiliev [144] has reviewed and listed the heat pipe $\mathrm{R} \& \mathrm{D}$ work on conventional heat pipes, heat pipe panels, loop heat pipes, vapor-dynamic thermosyphons, $\mathrm{micro} / \mathrm{miniature}$ heat pipes, and sorption heat pipes in different industrial applications. A novel approach is to utilize nanofluids to enhance the capabilities of heat pipes. Shafahi et al. [145] analyzed and modeled the influence of a nanofluid on the thermal performance of a cylindrical heat pipe. The authors reported that the nanoparticles within the liquid enhance the thermal performance of the heat pipe by reducing the thermal resistance while enhancing the maximum heat load it can carry. The existence of an optimum mass concentration for nanoparticles in maximizing the heat transfer limit and that the smaller particles have a more pronounced effect on the temperature gradient along the heat pipe was also established. Recently, Yau and Ahmadzadehtalatapeh [146] conducted a literature review on the application of horizontal heat pipe heat exchangers for air conditioning in tropical climates. Their work focused on the energy saving and dehumidification enhancement aspects of horizontal heat pipe heat exchangers. The related papers were grouped into three main categories and a summary of experimental and theoretical studies was presented.

A variation of the heat pipe called the "microheat pipe" (MHP) mostly used in electronic cooling applications was first proposed by Cotter [147]. Unlike conventional heat pipes, MHPs do not contain any wick structure, but instead consist of small noncircular (usually triangular) channels instead; the sharp-angled corner regions in these noncircular tubes serve as liquid return arteries. Microfluid flow channels in MHP have hydraulic diameters on the order of $10-500 \mu \mathrm{m}$. Smaller flow channels in MHP are desirable in order to achieve higher heat transfer coefficients and higher heat transfer surface area per unit flow volume. MHPs are also capable of removing large amounts of heat with the possibility of achieving extremely high heat fluxes near $1000 \mathrm{~W} / \mathrm{cm}^{2}$. Much research has been carried out in recent years to predict the performance of MHPs as evident in the excellent review papers of Vasiliev [148] and Sobhan et al. [149] on MHP research and development work.

Phase-change is also used in many other applications to enhance heat transfer as illustrated in the work of Khadrawi and Al-Nimr [150], who proposed and then analytically investigated a novel technique for the cooling of intermittently working internal combustion engines. This technique utilizes a phase change material, which absorbs heat to melt itself, and thus cools the engine as it runs, while the same phase change material releases heat upon restarting of the engine. The main findings of this work show that as the melting temperature and the enthalpy of melting increases the operational time of the system increases, especially for large values of melting temperature. The surface temperature when using a phase change material is much lower than that of conventional air cooling case.

3.6. Heat Transfer Enhancement Using Flexible Seals. Single layered (SL) and double layered (DL) microchannels supported by flexible seals are analyzed in the woks of Vafai and Khaled [151]. In their work, they related the deformation of the supporting seals to the average internal pressure by the theory of elasticity. This relation is coupled with the momentum equation which is solved numerically using an iterative implicit finite difference method. After solving these equations, they solved the energy equation. For the same flexible seals, they showed numerically that a flow that cause an expansion in the microchannel height by a factor of 1.5 causes a drop in the average surface temperature for SL by $53 \%$ times its value for rigid SL at the same pressure drop under the same constant heat flux. They showed that cooling effect due to hydrodynamic expansion increases as Prandtl number decreases. Further, their results show that SL flexible microchannel heat sinks mostly provide better cooling attributes compared to DL flexible microchannel heat sinks delivering the same coolant flow rate and having the same flexible seals. However, they showed that rigid DL microchannel heat sinks provides better cooling than rigid SL microchannel heat sinks when operated at the same pressure drop. Finally, they concluded that SL flexible microchannel heat sinks are preferred to be used for large pressure drop applications while DL flexible microchannel heat sinks are preferred to be utilized for applications involving low pressure drops along with stiff seals. Later on, Khaled [13] found out that the average temperature of the heated plate decreases as seals number $\left(F_{n}\right)$ increases until $F_{n}$ reaches an optimum after which this temperature starts to increase with an increase in $F_{n}$. 
3.6.1. Models for Heat Transfer Correlations. Khaled [152] later analyzed analytically two dimensional flexible thin film channel that has a small and variable height $h$ compared to its length $B$. The $x$-axis is taken along the coolant flow direction while $y$-axis is taken along its height. The width of the thin film channel, $D$, is assumed to be large enough such that twodimensional flow between the plates can be assumed. The height of the flexible thin film channel is considered to have the following generic form:

$$
\frac{h(x)}{h_{e}}=\left[\left(\frac{h_{i}}{h_{e}}\right)^{1 / n}-\left[\left(\frac{h_{i}}{h_{e}}\right)^{1 / n}-1\right]\left(\frac{x}{B}\right)\right]^{n},
$$

where $n, h_{e}$ and $h_{i}$ are the power-law index, exit, and inlet heights, respectively. When $n=1.0$, the inclination angle of the upper plate is uniform. As such, formula (36) with $n=1.0$ models the height profile for flexible thin film channels having inflexible plates. However, each differential element of the upper plate will have a different slope when $n \neq 1.0$. As such, formula (36) models height distributions of flexible thin film channels having flexible upper plates and fixed lower plates when $n \neq 1.0$. The case when $n=0.25$ mathematically represents the height profile when the upper plate stiffness is negligible compared to the seals stiffness. Recall that the seals stiffness is the applied tension force on the seal that is required to produce $1 \mathrm{~m}$ elongation in its thickness.

Khaled [152] showed that the maximum reduction in dimensionless heated plate temperature $\left(\theta_{W}\right)_{\text {AVG }}$ associated with case $n=0.25$ is $55 \%$ less than that for case with $n=1.0$ (when $H_{i}=h_{i} / h_{e}=3.0$ ). In addition, the maximum increase in wall shear stress for case with $n=0.25$ is $44 \%$ greater than that for case with $n=1.0$. The former percentage is greater than the latter one which again demonstrates the superiority of flexible thin film channels with flexible plates over those with inflexible plates. Finally, Khaled [13] developed a correlation for design purposes that relates $\left(\theta_{W}\right)_{\text {AVG }}$ to $H_{i}, n$, and Pee for the following range: $1.0<H_{i}<3.0,1.0<$ Pee $<50$ and $0.1<n<2.0$.

$$
\begin{aligned}
&\left(\theta_{W}\right)_{\mathrm{AVG}}=\frac{1}{\mathrm{Nu}_{\mathrm{AVG}}}+\frac{H_{i}^{(1-3 n) / n}-1}{2\left(H_{i}^{1 / n}-1\right)(1-3 n) \mathrm{Pe} \varepsilon}, \\
& \mathrm{Nu}_{\mathrm{AVG}}= 2.509+0.507 n+0.141(\mathrm{Pe} \varepsilon)-0.108 H_{i} \\
&-0.0415 n(\mathrm{Pe} \varepsilon)-0.630 n H_{i}+0.0904(\mathrm{Pe} \varepsilon) H_{i} \\
&+0.00356 n^{2}(\mathrm{Pe} \varepsilon)+0.00155 n(\mathrm{Pe} \varepsilon)^{2} \\
&+ 0.136 n^{2} H_{i}+0.0294 n H_{i}^{2}-0.00549(\mathrm{Pe} \varepsilon)^{2} H_{i} \\
&+ 0.00424(\mathrm{Pe} \varepsilon) H_{i}^{2}-4.21 \times 10^{-5} n^{2}(\mathrm{Pe} \varepsilon)^{2} H_{i}^{2} .
\end{aligned}
$$

The maximum percentage error between the results of the correlation and the numerical results is about $10 \%$. Where

$$
\begin{array}{cc}
\mathrm{Pe}=\frac{\rho c_{p} u_{o} h_{i}}{k} & \varepsilon=\frac{h_{e}}{B} \\
\theta_{w}=\frac{T_{W}-T_{i}}{q^{\prime \prime} h_{e} / k} & H_{i}=\frac{h_{i}}{h_{e}} .
\end{array}
$$

Note that $u_{o}$ is the average flow speed at exit. Note that Khaled [13] considered flexible thin films with heated lower plate with constant heat flux $\left(q^{\prime \prime}\right)$ and insulated upper plate.

3.7. Heat Transfer Enhancement Using Flexible Complex Seals. Khaled and Vafai [12] have also demonstrated that significant cooling inside flexible thin films including flexible microchannel heat sinks can be achieved if the supporting seals contain closed cavities which are in contact with the heated surface. They referred to this kind of sealing assembly as "flexible complex seals". For example, their results showed that expansion of microchannels of magnitude of 1.26 the initial height can cause a drop in the temperature of $16 \%$ the initial average heated plate temperature. Later on, Khaled [13] showed that the average temperature of the heated plate always decreases as thermal expansion parameter $\left(F_{T}\right)$ increases if thermal entry region is to be considered. While, it decreases as $F_{T}$ increases until $F_{T}$ reaches a critical value after which that temperature starts to increase for developed flows. Further, he showed that the decrease in the heated plate temperature is significant at lower values of Re, Pr and microchannel aspect ratio.

3.7.1. Models for Heat Transfer Correlations. Khaled and Vafai [153] generated the following correlation are for average Nusselt number $(\mathrm{Nu})_{\mathrm{AVG}}$ and the dimensionless average mean bulk temperature $\left(\theta_{m}\right)_{\mathrm{AVG}}$ for thin films supported by flexible complex seals with flexible upper plates for the specified range of parameters, $1.0<S_{1}<10,1.0<$ PeE $<50$ and $0<F_{T 1}<1.0$ :

$$
\begin{aligned}
& \mathrm{Nu}_{\mathrm{AVG}} \\
& =\frac{0.0455(\mathrm{Pe} \varepsilon)^{0.6793} F_{T 1}^{0.8345}+1.5285(\mathrm{Pe} \varepsilon)^{0.2877} S_{1}^{0.3225}}{\left(0.5169 K_{1}^{0.620}+1.255 \times 10^{-3} F_{T 1}^{1.8691}\right)^{0.5503}}, \\
& R^{2}=0.996, \\
& \left(\theta_{m}\right)_{\mathrm{AVG}} \quad \\
& =\frac{1.3709(\mathrm{Pe} \varepsilon)^{-0.7717} F_{T 1}^{1.6939}+1.1520(\mathrm{Pe \varepsilon})^{-0.9898} S_{1}^{-0.4380}}{\left(2.1144 S_{1}^{-0.3449}+1.8401 F_{T 1}^{1.3294}\right)^{2.4686}} \\
& R^{2}=0.992 .
\end{aligned}
$$

He considered a fixed lower plate of the thin film while the upper plate is flexible and separated from the lower plate by soft complex seals that allow a local expansion in the thin film heights due to both changes in internal pressure and the lower (heated) plate temperature. Similar effects are expected when the upper plate is a bimaterial plate separated from the lower plate via soft seals. He assumed that the thin film height varies linearly with local pressure and local lower plate temperature according to the following relationship:

$$
H(X)=\frac{h(x)}{h_{o}}=1+\frac{\bar{P}(X)}{S_{1}}+F_{T 1} \theta_{B}(X),
$$


where $F_{T 1}$ is the thermal expansion parameter which is equal to

$$
F_{T 1}=\beta \frac{q h_{\mathrm{o}}}{k} .
$$

The coefficient $\beta$ is thermal expansion coefficient of the flexible complex seals. The parameter $F_{T 1}$ increases as the heating load $q$, the thermal expansion coefficient $\beta$ and the reference thin film height increase while it decreases as the fluid thermal conductivity $k$ decreases. The stiffness parameter $S_{1}$ is related to the elastic properties of the flexible complex seals. $(\mathrm{Nu})_{\mathrm{AVG}}$ is defined as

$$
\begin{array}{r}
\mathrm{Nu}_{\mathrm{AVG}} \equiv \frac{\left(h_{c}\right)_{\mathrm{AVG}} h_{o}}{k}=\frac{1}{\left(\theta_{W}\right)_{\mathrm{AVG}}-\left(\theta_{m}\right)_{\mathrm{AVG}}}, \\
\left(\theta_{m, W}\right)_{\mathrm{AVG}}=\frac{\left(T_{m, W}\right)_{\mathrm{AVG}}-T_{1}}{q h_{o} / k} .
\end{array}
$$

3.8. Heat Transfer Enhancement Using Vortex Generators. Acharya et al. [155] conducted experiments using internally ribbed channel with cylindrical vortex generators placed above the ribs. They studied the effect of the spacing between the vortex generators and the ribs. They found that the heat and mass transfer depend on both the generator-rib spacing to rib height $(s / e)$ ratio and the Reynolds number. They showed that at low Reynolds number $(\operatorname{Re}=5000)$, the heat transfer enhancement was observed for all $s / e$ ratios. However, at high Reynolds number $(\operatorname{Re}=30,000)$, the enhancement was observed only for the largest $s / e$ ratio $(s / e=1.5)$. For this ratio, the generator wakes and rib shear layer interact with each other and promote mixing and thus, enhance heat transfer. For the smallest $s / e$ ratio $(s / e=$ $0.55)$, due to the smaller gap between the generator-ribs, at high Reynolds numbers the ribs act as a single element and prevent the redevelopment of the shear layer causing reduced heat transfer

Lin and Jang [156] numerically studied the performance of a wave-type vortex generator installed in a fin-tube heat exchanger. They found that an increase in the length or height of the vortex generator increases the heat transfer, as well as the friction losses. They reported up to $120 \%$ increase in the heat transfer coefficient at a maximum area reduction of $20 \%$, accompanied by a $48 \%$ increase in the friction factor.

Tiwari et al. [157] numerically simulated the effect of the delta winglet type vortex generator on the flow and heat transfer in a rectangular duct with a built-in circular tube. They observed that the vortices induced by the vortex generator resulted in an increase in the span-averaged Nusselt number at the trailing edge of the vortex generator by a factor of 2.5 and the heat transfer enhancement of $230 \%$ in the near wake region.

Dupont et al. [158] investigated the flow in an industrial plate-fin heat exchanger with periodically arranged vortex generators for a range of Reynolds number varying from 1000 to 5000 . They found that the vortex intensity increases with the Reynolds number.

O'Brien et al. [159] conducted experimental study in a narrow rectangular duct fitted with an elliptical tube inside a fin tube heat exchanger, for a range of Reynolds number varying from 500 to 6300 . A pair of delta winglets was used as the vortex generator. They estimated the local surface heat transfer coefficient and pressure drop. They found that the addition of a single winglet pair could increase the heat transfer by $38 \%$. They also found that the increase in the friction factor due to the addition of a winglet pair was less than $10 \%$ over the range of Reynolds numbers studied.

Tsay et al. [160] numerically investigated the heat transfer enhancement due to a vertical baffle in a backward-facing step flow channel. The effect of the baffle height, thickness, and the distance between the baffle and the backward facing step on the flow structure was studied in detail for a range of Reynolds number varying from 100 to 500 . They found that an introduction of a baffle into the flow could increase the average Nusselt number by $190 \%$. They also observed that the flow conditions and heat transfer characteristics are strong function of the baffle position

Joardar and Jacobi [161] carried out experimental investigations to evaluate the effectiveness of delta-wing type vortex generators by full-scale wind-tunnel testing of a compact heat exchanger typical to those used in automotive systems. The mechanisms important to vortex enhancement methods are discussed, and a basis for selecting a deltawing design as a vortex generator is established. The heat transfer and pressure drop performance are assessed at full scale under both dry- and wet-surface conditions for a louvered-fin baseline and for a vortex-enhanced louveredfin heat exchanger. An average heat transfer increase over the baseline case of $21 \%$ for dry conditions and $23.4 \%$ for wet conditions was achieved with a pressure drop penalty smaller than $7 \%$. Vortex generation is proven to provide an improved thermal-hydraulic performance in compact heat exchangers for automotive systems.

Heat transfer enhancement in a heat exchanger tube by installing a baffle is reported by Nasiruddin and Siddiqui [162]. They conducted a detailed numerical investigation of the vortex generator design and its impact on the heat transfer enhancement in a heat exchanger tube. The effect of baffle size and orientation on the heat transfer enhancement was studied in detail. Three different baffle arrangements were considered. The results show that for the vertical baffle, an increase in the baffle height causes a substantial increase in the Nusselt number but the pressure loss is also very significant. For the inclined baffles, the results show that the Nusselt number enhancement is almost independent of the baffle inclination angle, with the maximum and average Nusselt number $120 \%$ and $70 \%$ higher than that for the case of no baffle, respectively. For a given baffle geometry, the Nusselt number enhancement is increased by more than a factor of two as the Reynolds number decreased from 20,000 to 5000 . Simulations were conducted by introducing another baffle to enhance heat transfer. The results show that the average Nusselt number for the two baffles case is $20 \%$ higher than the one baffle case and $82 \%$ higher than the no baffle case. The above results suggest that a significant heat transfer enhancement in a heat exchanger tube can be achieved by introducing a baffle inclined towards the downstream side, with the minimum pressure loss. 
TABle 3: Constants (a)-(d) for formula (45) along with the Ranges of the Parameters [154].

\begin{tabular}{|c|c|c|c|c|}
\hline$H(m)$ & $a$ & $b$ & $c$ & $D$ \\
\hline 0.125 & 1.51654 & 0.3471 & 0.1081 & -0.4079 \\
\hline \multirow[t]{2}{*}{0.250} & 1.3951 & 0.3144 & 0.4538 & 0.0278 \\
\hline & & $\frac{\rho_{f} u_{m} H}{\mu_{f}} \leq 2000$ & $0.125 \leq w(m) \leq 0.5$ & $1 \leq \frac{k_{s}}{k_{f}} \leq 6600$ \\
\hline
\end{tabular}

Delta winglets are known to induce the formation of stream wise vortices and increase heat transfer between a working fluid and the surface on which the winglets are placed. Lawson and Thole [163] employed delta winglets to augment heat transfer on the tube surface of louvered fin heat exchangers. It is shown that delta winglets placed on louvered fins produce augmentations in heat transfer along the tube wall as high as $47 \%$ with a corresponding increase of $19 \%$ in pressure losses. Manufacturing constraints are considered in this study, whereby piercings in the louvered fins resulting from stamping the winglets into the louvered fins are simulated. Comparisons of measured heat transfer coefficients with and without piercings indicate that piercings reduce average heat transfer augmentations, but significant increases still occur with respect to no winglets present.

Air-side heat transfer and friction characteristics of five kinds of fin-and-tube heat exchangers, with the number of tube rows $(N=12)$ and the diameter of tubes $\left(D_{o}=18 \mathrm{~mm}\right)$, have been experimentally investigated by Tang et al. [164]. The test samples consist of five types of fin configurations: crimped spiral fin, plain fin, slit fin, fin with delta-wing longitudinal vortex generators (VGs), and mixed fin with front 6-row vortex-generator fin and rear 6-row slit fin. The heat transfer and friction factor correlations for different types of heat exchangers were obtained with the Reynolds numbers ranging from 4000 to 10000 . It was found that crimped spiral fin provides higher heat transfer and pressure drop than the other four fins. The air-side performance of heat exchangers with the above five fins has been evaluated under three sets of criteria and it was shown that the heat exchanger with mixed fin (front vortex-generator fin and rear slit fin) has better performance than that with fin with delta-wing vortex generators, and the slit fin offers best heat transfer performance at high Reynolds numbers. Based on the correlations of numerical data, Genetic Algorithm optimization was carried out, and the optimization results indicated that the increase of VG attack angle or length, or decrease of VG height may enhance the performance of vortex-generator fin. The heat transfer performances for optimized vortex-generator fin and slit fin at hand have been compared with numerical method.

A systematic numerical study of the effects of heat transfer and pressure drop produced by vortex promoters of various shapes in a $2 \mathrm{D}$, laminar flow in a microchannel have been presented by Meis et al. [168]. The liquid is assumed to be water, with temperature dependent viscosity and thermal conductivity. It is intended to obtain useful design criteria of microcooling systems, taking into account that practical solutions should be both thermally efficient and not expensive in terms of the pumping power. Three reference cross sections, namely circular/elliptical, rectangular, and triangular, at various aspect ratios are considered. The effect of the blockage ratio, the Reynolds number, and the relative position and orientation of the obstacle are also studied. Some design guidelines based on two figures of merit (related to thermal efficiency and pressure drop, respectively), which could be used in an engineering environment are provided

Sheik Ismail et al. [169] have presented a review of research and developments of compact offset and wavy platefin heat exchangers. The review has been summarized under three major sections. They are offset fin characteristics, wavy fin characteristics and nonuniformity of the inlet fluid flow. The various research aspects relating to internal single phase flow studied in offset and wavy fins by the researchers are compared and summarized. Further, the works done on the nonuniformity of this fluid flow at the inlet of the compact heat exchangers are addressed and the methods available to minimize these effects are compared.

3.8.1. Models for Heat Transfer and Friction Factor Correlations. Recently, Eiamsa-ard et al. [170] have experimentally investigated the heat transfer, flow friction and thermal performance factor characteristics in a tube fitted with deltawinglet twisted tape, using water as working fluid. Influences of the oblique delta-winglet twisted tape (O-DWT) and straight delta-winglet twisted tape (S-DWT) arrangements are also described. The experiments are conducted using the tapes with three twist ratios $(y / w=3,4$ and 5) and three depth of wing cut ratios $(D R=d / w=0.11,0.21$ and 0.32 ) over a Reynolds number (Re) range of 3000-27,000 in a uniform wall heat flux tube. Note that $d, y$, and $w$ are the depth of wing cut, the twisted tape pitch and the tape width, respectively. The obtained results show that mean Nusselt number and mean friction factor in the tube with the deltawinglet twisted tape increase with decreasing twisted ratio $(y / w)$ and increasing depth of wing cut ratio $(D R)$. It is also observed that the O-DWT is more effective turbulator giving higher heat transfer coefficient than the S-DWT. Over the range considered, Nusselt number, friction factor and thermal performance factor in a tube with the O-DWT are, respectively, 1.04 to $1.64,1.09$ to 1.95 , and 1.05 to 1.13 times of those in the tube with typical twisted tape (TT).

Empirical correlations for Nusselt number $(\mathrm{Nu})$, friction factor $(f)$, thermal performance factor $(\eta)$ are developed for the tube with delta-winglet twisted tape inserts in the range of Re between 3000 and 27,000, $\operatorname{Pr}=4.91-5.57$, twist 
ratio $(y / w=3,4$ and 5$)$, and depth of wing cut ratios $(D R=d / w=0.11,0.21$ and 0.32$)$ as follows. The predicted data are within $\pm 10 \%$ for Nusselt number and $\pm 10 \%$ for friction factor. They are the following for oblique deltawinglet twisted tapes:

$$
\begin{gathered}
\mathrm{Nu}=0.18 \operatorname{Re}^{0.67} \operatorname{Pr}^{0.4}\left(\frac{y}{w}\right)^{-0.423}\left(1+\frac{d}{w}\right)^{0.962}, \\
f=24.8 \operatorname{Re}^{-0.51}\left(\frac{y}{w}\right)^{-0.566}\left(1+\frac{d}{w}\right)^{1.87}, \\
\eta=2.04 \operatorname{Re}^{-0.042} \operatorname{Pr}^{0.4}\left(\frac{y}{w}\right)^{-0.261}\left(1+\frac{d}{w}\right)^{0.45},
\end{gathered}
$$

while they are the following for straight delta-winglet twisted tapes:

$$
\begin{gathered}
\mathrm{Nu}=0.184 \operatorname{Re}^{0.675} \operatorname{Pr}^{0.4}\left(\frac{y}{w}\right)^{-0.465}\left(1+\frac{d}{w}\right)^{0.76} \\
f=21.7 \operatorname{Re}^{-0.45}\left(\frac{y}{w}\right)^{-0.564}\left(1+\frac{d}{w}\right)^{1.41}, \\
\eta=2.164 \operatorname{Re}^{-0.0435} \operatorname{Pr}^{0.4}\left(\frac{y}{w}\right)^{-0.304}\left(1+\frac{d}{w}\right)^{0.356}
\end{gathered}
$$

3.9. Heat Transfer Enhancement Using Protrusions. The effect of repeated horizontal protrusions on the free-convection heat transfer in a vertical, asymmetrically heated, channel has been experimentally investigated by Tanda [171]. The protrusions have a square section and are made of a lowthermal-conductivity material. Experiments were conducted by varying the number of the protrusions over the heated surface (whose height was held fixed) and the aspect ratio of the channel. The convective fluid was air and the wallto-ambient air temperature difference was set equal to $45 \mathrm{~K}$. The local heat transfer coefficient was obtained by means of the schlieren optical technique. The protrusions were found to significantly alter the heat transfer distribution along the heated surface of the channel, especially in the vicinity of each obstacle. For the ranges of parameters studied, the addition of low-conductivity protrusions leads to a decrease in the average heat transfer coefficient, as compared to that for the smooth surface, in the $0-7 \%$ range for the largest channel aspect ratio and in the $18-43 \%$ for the smallest channel aspect ratio.

Saidi and Sundén [172] have conducted a numerical analysis of the instantaneous flow and heat transfer has been carried out for offset strip fin geometries in selfsustained oscillatory flow. The analysis is based on the twodimensional solution of the governing equations of the fluid flow and heat transfer with the aid of appropriate computational fluid dynamics methods. Unsteady calculations have been carried out. The obtained time-dependent results are compared with previous numerical and experimental results in terms of mean values, as well as oscillation characteristics. The mechanisms of heat transfer enhancement are discussed and it has been shown that the fluctuating temperature and velocity second moments exhibit non-zero values over the fins. The creation processes of the temperature and velocity fluctuations have been studied and the dissimilarity between these has been proved.

Jubran et al. [173] investigated experimentally the effects of rectangular and noncubical obstacles of various lengths, widths and heights on pressure drop and heat transfer enhancements. They found that changes in obstacle size or shape can lead to Nusselt number increases as height as $40 \%$. Sparrow et al. [165, 166] found out that mass transfer enhancements of up to $100 \%$ can be obtained using perturbations of uniform arrays of square obstacles. An extensive investigation of the fluid and heat transfer in a parallel plate channel with a solid conducting obstacle is conducted by Young and Vafai [154]. The rectangular obstacle was found to change the parabolic velocity field significantly resulting in recirculation zones both up- and downstream and a thermal boundary layer along the top face. Their results show that the shape and material of the obstacle have significant effects on the fluid flow and heat transfer.

3.9.1. Models for Heat Transfer Correlations. Young and Vafai [154] proposed Correlation for the obstacle mean Nusselt numbers were found to describe the numerical results with mean errors less than $6 \%$. The correlations have the following functional form:

$$
\overline{\mathrm{Nu}_{m}}=\frac{h_{m} H}{k_{f}}=a \operatorname{Re}_{D_{h}}^{b}\left(\frac{k_{s} / k_{f}}{c+k_{s} / k_{f}}\right)(1+w)^{d},
$$

where $h_{m}, H, k_{s}, k_{f}$, and $w$ are the average convection heat transfer coefficient over the whole obstacle surface, channel height, obstacle thermal conductivity, fluid thermal conductivity, and the obstacle length along the flow direction, respectively. The constants $a, b, c$ and $d$ are found from Table 3 along with the range of the parameters where the inlet length before the obstacle is long enough such that the flow before the obstacle is fully developed. The length of the channel after the obstacle is considered long enough such that the recirculation zones down stream after the obstacle reattached well ahead before the channel outlet. Note that $u_{m}$ is the mean flow velocity inside the channel. The correlation is based on insulated lower and upper channel boundaries and that the heat flux at the lower obstacle surface is constant $\left(q^{\prime \prime}\right)$ where it is equal to $q^{\prime \prime} w=h_{m}(2 h+w)\left(\bar{T}_{w}-\right.$ $\left.T_{e}\right)$. In another work, Young and Vafai [174] performed a comprehensive numerical investigation of fluid and thermal transport within two-dimensional channel containing large arrays of heated obstacles. They found that widely spaced obstacles can effectively transfer thermal energy into the fluid. They studied the effect of the periodicity of the obstacles on heat transfer by doubling the number of obstacles and evaluating the mean Nusslet numbers. The mean Nusselt number was found to reach $5 \%$ and $10 \%$ difference levels, referenced to ninth obstacle, at the eighth and the seventh obstacles, respectively. The case with porous inserts have been discussed in the works of Alkam et al. [175].

\subsection{Heat Transfer Enhancement Using Ultra High Thermal Conductivity Composite Materials. Composite materials have}


TABLE 4: Estimated the highest recorded heat transfer enhancement level due to each enhancer.

\begin{tabular}{lr}
\hline Heat Transfer Enhancer Type & $\left(\frac{\text { Heat transfer due presence of the enhancer }}{\text { Heat transfer in absence of the enhancer }}\right)$ \\
\hline Fins inside tubes & $2.0,[37]$ \\
Microfins inside tubes & 1.0 for laminar, [70] for turbulent, [70] \\
& $\approx 12.0\left(k_{\text {eff }} / k_{f}\right),[78]$ \\
Porous media & $3.5,[10]$ \\
Nanofluids & $2.0,[151]$ \\
Flexible seals & $3.0,[153]$ \\
Flexible complex seals & $2.5,[157]$ \\
Vortex generators & $2.0,[165,166]$ \\
Protrusions & $6,[167]$ \\
Ultra high thermal conductivity composite materials &
\end{tabular}

been used primarily for structural applications. However, they have been found to be useful for heat dissipation especially in electronic devices. An example of such these material is the metal matrix composite (MMC). Typical MMCs that includes aluminum and copper matrix composites do not show substantial improvements in thermal conductivity except when reinforcing agent of vapor grown carbon fiber (VGCF) is used as shown in the work of Ting and Lake [176]. For example, VGCF-reinforced aluminum matrix composite exhibits a thermal conductivity that can be $642 \mathrm{~W} / \mathrm{mK}$ with a density of $2440 \mathrm{~kg} / \mathrm{m}^{3}$ using $36.5 \%$ of VGCF. However, all MMCs are electrically conductive. Chen and Teng [167] have shown that VGCF mat reinforced epoxy composites can have thermal conductivities larger $695 \mathrm{~W} / \mathrm{mK}$ with density of $1480 \mathrm{~kg} / \mathrm{m}^{3}$ in addition of having an electrically insulating surface. This is with a reinforcement of $56 \%$ by volume of heat treated VGCF. Recently, Naito et al. [177] have shown that grafting of high thermal conductivity carbon nanotubes (CNTs) is very effective in improving the thermal conductivity of certain types of carbon fibers, which can reach to $47 \%$ improvement.

\section{Conclusions}

In this paper, the following heat transfer enhancers are described and reviewed: (a) extended surfaces including fins and microfins, (b) porous media, (c) large particles suspensions, (d) nanofluids, (e) phase-change devices, (f) flexible seals, (g) flexible complex seals, (h) vortex generators, (i) protrusions, and (j) ultra high thermal conductivity composite materials. Different research works about each one have been reviewed and many methods that assist their enhancement effects have been extracted from the literature. Among of these methods presented in the literature are using joint-fins, fin roots, fin net works, biconvections, permeable fins, porous fins, helical microfins, and using complicated designs of twisted tapes. It was concluded that more attention should be made towards single phase heat transfer augmented with microfins in order to alleviate the disagreements between the works of the different authors. Also, it was found that additional attention should be made towards uncovering the main mechanisms of heat transfer enhancements due to the presence of nanofluids. Moreover, we concluded that perhaps the successful modeling of flow and heat transfer inside porous media, which is a wellrecognized passive enhancement method, could help in well discovering the mechanism of heat transfer enhancements due to nanofluids. This is due to some similarities between both media. In addition, it is concluded that noticeable attentions from researchers are required towards further modeling flow and heat transfer inside convective media supported by flexible/flexible-complex seals in order too compute their levels of heat transfer enhancements. Eventually, many recent works related to passive augmentations of heat transfer using vortex generators, protrusions, and ultra high thermal conductivity composite material have been reviewed. Finally, the estimated maximum levels of the heat transfer enhancement due to each enhancer described in this report were presented in Table 4 .

\section{Acknowledgment}

The authors acknowledge the full support of this work by King Abdulaziz City for Science and Technology (KACST) under project no. 8-ENE192-3.

\section{References}

[1] A. E. Bergles, Handbook of Heat Transfer, McGraw-Hill, New York, NY, USA, 3rd edition, 1998.

[2] A. E. Bergles, "The implications and challenges of enhanced heat tranfer for the chemical process industries," Chemical 
Engineering Research and Design, vol. 79, no. 4, pp. 437-444, 2001.

[3] E. I. Nesis, A. F. Shatalov, and N. P. Karmatskii, "Dependence of the heat transfer coefficient on the vibration amplitude and frequency of a vertical thin heater," Journal of Engineering Physics and Thermophysics, vol. 67, no. 1-2, pp. 696-698, 1994.

[4] J. K. Hagge and G. H. Junkhan, "Experimental study of a method of mechanical augmentation of convective heat transfer in air," Tech. Rep. HTL3, ISU-ERI-Ames-74158, Iowa State University, Amsterdam, The Netherlands, 1975.

[5] H. M. Li, K. S. Ye, Y. K. Tan, and S. J. Deng, "Investigation on tube-side flow visualization, friction factors and heat transfer characteristics of helical-ridging tubes," in Proceedings of the 7th International Heat Transfer Conference, vol. 3, pp. 75-80, Munich, Germany, 1982.

[6] J. A. Kohler and K. E. Staner, "High performance heat transfer surfaces," in Handbook of Applied Thermal Design, E. C. Guyer, Ed., pp. 7.37-7.49, McGraw-Hill, New York, NY, USA, 1984.

[7] B. Arman and T. J. Rabas, "Disruption share effects on the performance of enhanced tubes with the separation and reattachment mechanism," in Proceedings of the 28th National Heat Transfer Conference and Exhibition, vol. 202, pp. 67-75, August 1992.

[8] A. Amiri and K. Vafai, "Analysis of dispersion effects and non-thermal equilibrium, non-Darcian, variable porosity incompressible flow through porous media," International Journal of Heat and Mass Transfer, vol. 37, no. 6, pp. 939-954, 1994.

[9] A. A. Mohamad, "Heat transfer enhancements in heat exchangers fitted with porous media. Part I: constant wall temperature," International Journal of Thermal Sciences, vol. 42, no. 4, pp. 385-395, 2003.

[10] Y. Ding, H. Alias, D. Wen, and R. A. Williams, "Heat transfer of aqueous suspensions of carbon nanotubes (CNT nanofluids)," International Journal of Heat and Mass Transfer, vol. 49, no. 1-2, pp. 240-250, 2006.

[11] A.-R. A. Khaled and K. Vafai, "Flow and heat transfer inside thin films supported by soft seals in the presence of internal and external pressure pulsations," International Journal of Heat and Mass Transfer, vol. 45, no. 26, pp. 5107-5115, 2002.

[12] A.-R. A. Khaled and K. Vafai, "Cooling enhancements in thin films supported by flexible complex seals in the presence ultrafine suspensions," Journal of Heat Transfer, vol. 125, no. 5, pp. 916-925, 2003.

[13] A.-R. A. Khaled, "The role of expandable thermal systems in improving performance of thermal devices," International Journal of Thermal Sciences, vol. 46, no. 4, pp. 413-418, 2007.

[14] A.-R. A. Khaled, "Heat transfer analysis through solar and rooted fins," Journal of Heat Transfer, vol. 130, no. 7, Article ID 074503, 2008.

[15] W. M. Kays, "Pin-fin heat-exchanger surfaces," Journal of Heat Transfer, vol. 77, pp. 471-483, 1955.

[16] D. O. Kern and A. D. Kraus, Extended Surface Heat Transfer, McGraw-Hill, New York, NY, USA, 1972.

[17] A. D. Kraus, "Sixty-five years of extended surface technology (1922-1987)," Applied Mechanical Review, vol. 41, pp. 621$364,1988$.

[18] P. J. Schenider, Conduction Heat Transfer, Addison Wesley, Reading, Mass, USA, 1955.
[19] A. D. Kraus, A. Aziz, and J. R. Welty, Extended Surface Heat Transfer, John Wiley \& Sons, New York, NY, USA, 2001.

[20] S. Kakaç and H. Liu, Heat Exchangers: Selection, Rating, and Thermal Design, CRC Press, Boca Raton, Fla, USA, 2001.

[21] N. Sahiti, A. Lemouedda, D. Stojkovic, F. Durst, and E. Franz, "Performance comparison of pin fin in-duct flow arrays with various pin cross-sections," Applied Thermal Engineering, vol. 26, no. 11-12, pp. 1176-1192, 2006.

[22] J. C. Choi and S. D. Kim, "Heat-transfer characteristics of a latent heat storage system using $\mathrm{MgCl}_{2} \cdot 6 \mathrm{H}_{2} \mathrm{O}$," Energy, vol. 17, no. 12, pp. 1153-1164, 1992.

[23] B. Horbaniuc, G. Dumitrascu, and A. Popescu, "Mathematical models for the study of solidification within a longitudinally finned heat pipe latent heat thermal storage system," Energy Conversion and Management, vol. 40, no. 15, pp. 1765-1774, 1999.

[24] Y. Zhang and A. Faghri, "Heat transfer enhancement in latent heat thermal energy storage system by using the internally finned tube," International Journal of Heat and Mass Transfer, vol. 39, no. 15, pp. 3165-3173, 1996.

[25] F. Agyenim, P. Eames, and M. Smyth, "A comparison of heat transfer enhancement in a medium temperature thermal energy storage heat exchanger using fins," Solar Energy, vol. 83, no. 9, pp. 1509-1520, 2009.

[26] A. Nuntaphan, T. Kiatsiriroat, and C. C. Wang, "Air side performance at low Reynolds number of cross-flow heat exchanger using crimped spiral fins," International Communications in Heat and Mass Transfer, vol. 32, no. 1-2, pp. 151165, 2005.

[27] R. M. Manglik and A. E. Bergles, "Swirl flow heat transfer and pressure drop with twisted-tape inserts," Advances in Heat Transfer, vol. 36, article 183, 2002.

[28] A. Alebrahim and A. Bejan, "Constructal trees of circular fins for conductive and convective heat transfer," International Journal of Heat and Mass Transfer, vol. 42, no. 19, pp. 35853597, 1999.

[29] M. Almogbel and A. Bejan, "Cylindrical trees of pin fins," International Journal of Heat and Mass Transfer, vol. 43, no. 23, pp. 4285-4297, 2000.

[30] A.-R. A. Khaled, "Heat transfer enhancement in hairy fin systems," Applied Thermal Engineering, vol. 27, no. 1, pp. 250-257, 2007.

[31] A.-R. A. Khaled, "Maximizing heat transfer through joint fin systems," Journal of Heat Transfer, vol. 128, no. 2, pp. 203206, 2006.

[32] A.-R. A. Khaled, "Analysis of heat transfer through Biconvection fins," International Journal of Thermal Sciences, vol. 48, no. 1, pp. 122-132, 2009.

[33] S. Kiwan and M. A. Al-Nimr, "Using porous fins for heat transfer enhancement," Journal of Heat Transfer, vol. 123, no. 4, pp. 790-795, 2001.

[34] A.-R. A. Khaled, "Investigation of heat transfer enhancement using permeable fins," Journal of Heat Transfer, vol. 132, Article ID 034503, 2010.

[35] S. Al-Fahed, L. M. Chamra, and W. Chakroun, "Pressure drop and heat transfer comparison for both microfin tube and twisted-tape inserts in laminar flow," Experimental Thermal and Fluid Science, vol. 18, no. 4, pp. 323-333, 1998.

[36] B. Shome and M. K. Jensen, "Experimental investigation of laminar flow and heat transfer in internally finned tubes," Journal of Enhanced Heat Transfer, vol. 4, no. 1, pp. 53-70, 1996. 
[37] W. E. Hilding and C. H. Coogan, "Heat transfer and pressure loss measurements in internally finned tubes," in Proceedings of ASME Symposium on Air Cooled Heat Exchangers, pp. 57$85,1964$.

[38] E. K. Kalinin and S. A. Yarkho, "The effect of Reynolds and Prandtl numbers on the effectiveness of heat transfer enhancement in pipes," Inzhenerno-Fizicheskii Zhurnal, vol. 11, no. 4, pp. 426-431, 1966.

[39] Y. A. Vasilchenko and M. S. Barbaritskaya, "Resistance with non-isothermal fluid flow in tubes with longitudinal fins," Thermal Engineering, vol. 16, no. 1, pp. 28-35, 1969.

[40] Y. A. Vasilchenko and M. S. Barbaritskaya, "Heat transfer in tubes with longitudinal fins," Thermal Engineering, vol. 16, no. 1, pp. 105-109, 1969.

[41] A. E. Bergles, G. S. Brown, and W. D. Sinder, "Heat- transfer performance of internally finned tubes," ASME Paper no. 71HT-31, pp. 1-7, 1971.

[42] A. P. Watkinson, D. L. Miletti, and P. Tarassoff, "Turbulent heat transfer and pressure drop in internally finned tubes," AIChE Symposium Series, vol. 69, no. 131, pp. 94-103, 1973.

[43] A. P. Watkinson, D. L. Miletti, and G. R. Kubanek, "Heat transfer and pressure drop of internally finned tubes in turbulent air flow," ASHRAE Transactions, vol. 81, part 1, pp. 330-347, 1975, paper no. 2347.

[44] T. C. Carnavos, "Cooling air in turbulent flow with internally finned tubes," in Proceedings of the 17th National Heat Transfer Conference, 1977, AIChE paper 4.

[45] T. C. Carnavos, "Heat transfer performance of internally finned tubes in turbulent flow," Heat Transfer Engineering, vol. 1, no. 4, pp. 32-37, 1980.

[46] R. D. Armstrong and A. E. Bergles, "Enhanced ceramic tubes for high temperature waste heat recovery," Heat Transfer Laboratory Report HTL-1, Rensselaer Polytechnic Institute, 1989.

[47] M. B. Pate, Z. H. Ayub, and J. Kohler, "Heat exchangers for the air-conditioning and refrigeration industry," in Compact Heat Exchangers-A Festschrift for A. L. London, R. K. Shah, A. D. Kraus, and D. Metzger, Eds., pp. 567-590, Hemisphere, Washington, DC, USA, 1990.

[48] T. S. Ravigururajan and T. J. Rabas, "Turbulent flow in integrally enhanced tubes, Part 1: comprehensive review and database development," Heat Transfer Engineering, vol. 17, no. 2, pp. 19-29, 1996.

[49] J. B. Copetti, M. H. Macagnan, D. De Souza, and R. de Césaro Oliveski, "Experiments with micro-fin tube in single phase," International Journal of Refrigeration, vol. 27, no. 8, pp. 876$883,2004$.

[50] L. J. Brognaux, R. L. Webb, L. M. Chamra, and B. Y. Chung, "Single-phase heat transfer in micro-fin tubes," International Journal of Heat and Mass Transfer, vol. 40, no. 18, pp. 43454357, 1997.

[51] L. M. Schlager, M. B. Pate, and A. E. Bergles, "Heat transfer and pressure drop during evaporation and condensation of R22 in horizontal micro-fin tubes," International Journal of Refrigeration, vol. 12, no. 1, pp. 6-14, 1989.

[52] J. C. Khanpara, A. E. Bergles, and M. B. Pate, "Augmentation of R-113 in-tube evaporation with micro-fin tubes," ASHRAE Transactions, vol. 92, part 2, pp. 506-524, 1986.

[53] J. C. Khanpara, M. B. Pate, and A. E. Bergles, "Local evaporation heat transfer in a smooth tube and a micro-fin tube using refrigerants 22 and 113," in Proceedings of Boiling and Condensation in Heat Transfer Equipment, pp. 31-39, 1987, HTD-85.
[54] S. F. Al-Fahed, Z. H. Ayub, A. M. Al-Marafie, and B. M. Soliman, "Heat transfer and pressure drop in a tube with internal microfins under turbulent water flow conditions," Experimental Thermal and Fluid Science, vol. 7, no. 3, pp. 249-253, 1993.

[55] C. B. Chiou, C. C. Wang, and D. C. Lu, "Single-phase heat transfer and pressure drop characteristics of microfin tubes," in Proceedings of the ASHRAE Annual Meeting, pp. 10411048, June 1995, no. SD-95-11-1.

[56] M. Huq, A. M. Aziz-ul Huq, and M. M. Rahman, "Experimental measurements of heat transfer in an internally finned tube," International Communications in Heat and Mass Transfer, vol. 25, no. 5, pp. 619-630, 1998.

[57] M. K. Jensen and A. Vlakancic, "Experimental investigation of turbulent heat transfer and fluid flow in internally finned tubes," International Journal of Heat and Mass Transfer, vol. 42, no. 7, pp. 1343-1351, 1999.

[58] R. L. Webb, R. Narayanamurthy, and P. Thors, "Heat transfer and friction characteristics of internal helical-rib roughness," Journal of Heat Transfer, vol. 122, no. 1, pp. 134-142, 2000.

[59] H. S. Wang and J. W. Rose, "Prediction of effective friction factors for single-phase flow in horizontal microfin tubes," International Journal of Refrigeration, vol. 27, no. 8, pp. 904913, 2004.

[60] D. H. Han and K.-J. Lee, "Single-phase heat transfer and flow characteristics of micro-fin tubes," Applied Thermal Engineering, vol. 25, no. 11-12, pp. 1657-1669, 2005.

[61] X.-W. Li, J.-A. Meng, and Z.-X. Li, "Experimental study of single-phase pressure drop and heat transfer in a micro-fin tube," Experimental Thermal and Fluid Science, vol. 32, no. 2, pp. 641-648, 2007.

[62] G. J. Zdaniuk, L. M. Chamra, and D. Keith Walters, "Correlating heat transfer and friction in helically-finned tubes using artificial neural networks," International Journal of Heat and Mass Transfer, vol. 50, no. 23-24, pp. 4713-4723, 2007.

[63] G. J. Zdaniuk, L. M. Chamra, and P. J. Mago, "Experimental determination of heat transfer and friction in helically-finned tubes," Experimental Thermal and Fluid Science, vol. 32, no. 3, pp. 761-775, 2008.

[64] M. Siddique and M. Alhazmy, "Experimental study of turbulent single-phase flow and heat transfer inside a microfinned tube," International Journal of Refrigeration, vol. 31, no. 2, pp. 234-241, 2008.

[65] V. Gnielinski, "New equations for heat and mass transfer in turbulent pipe and channel flow," International Chemical Engineering, vol. 16, pp. 359-368, 1976.

[66] S. E. Haaland, "Simple and explicit formulas for the friction factor in turbulent pipe flow," Journal of Fluids Engineering, vol. 105, no. 1, pp. 89-90, 1983.

[67] G. J. Zdaniuk, R. Luck, and L. M. Chamra, "Linear correlation of heat transfer and friction in helically-finned tubes using five simple groups of parameters," International Journal of Heat and Mass Transfer, vol. 51, no. 13-14, pp. 3548-3555, 2008.

[68] R. L. Webb, "Single-phase heat transfer, friction, and fouling characteristics of three-dimensional cone roughness in tube flow," International Journal of Heat and Mass Transfer, vol. 52, no. 11-12, pp. 2624-2631, 2009.

[69] W. Li and R. L. Webb, "Fouling in enhanced tubes using cooling tower water. Part II: combined particulate and precipitation fouling," International Journal of Heat and Mass Transfer, vol. 43, no. 19, pp. 3579-3588, 2000. 
[70] P. Bharadwaj, A. D. Khondge, and A. W. Date, "Heat transfer and pressure drop in a spirally grooved tube with twisted tape insert," International Journal of Heat and Mass Transfer, vol. 52, no. 7-8, pp. 1938-1944, 2009.

[71] S. Kiwan and O. Zeitoun, "Natural convection in a horizontal cylindrical annulus using porous fins," International Journal of Numerical Methods for Heat and Fluid Flow, vol. 18, no. 5, pp. 618-634, 2008.

[72] T. K. Aldoss, M. A. Al-Nimr, and M. A. Hader, "Using capsulated liquid metal fins for heat transfer enhancement," Journal of Enhanced Heat Transfer, vol. 11, no. 2, pp. 151-160, 2004.

[73] D. F. Dipprey and R. H. Sabersky, "Heat and momentum transfer in smooth and rough tubes at various prandtl numbers," International Journal of Heat and Mass Transfer, vol. 6, no. 5, pp. 329-353, 1963.

[74] G. Lauriat and R. Ghafir, "Forced convective transfer in porous media," in Handbook of Porous Media, K. Vafai and H. A. Hadim, Eds., Marcel Dekker, New York, NY, USA, 2000.

[75] J. Y. Jang and J. L. Chen, "Forced convection in a parallel plate channel partially filled with a high porosity medium," International Communications in Heat and Mass Transfer, vol. 19, no. 2, pp. 263-273, 1992.

[76] S. Chikh, A. Boumedien, K. Bouhadef, and G. Lauriat, "Analytical solution of non-Darcian forced convection in an annular duct partially filled with a porous medium," International Journal of Heat and Mass Transfer, vol. 38, no. 9, pp. 1543-1551, 1995.

[77] S. Chikh, A. Boumedien, K. Bouhadef, and G. Lauriat, "NonDarcian forced convection analysis in an annulus partially filled with a porous material," Numerical Heat Transfer A, vol. 28, no. 6, pp. 707-722, 1995.

[78] M. A. Al-Nimr and M. K. Alkam, "Unsteady non-Darcian forced convection analysis in an annulus partially filled with a porous material," Journal of Heat Transfer, vol. 119, no. 4, pp. 799-804, 1997.

[79] M. K. Alkam and M. A. Al-Nimr, "Improving the performance of double-pipe heat exchangers by using porous substrates," International Journal of Heat and Mass Transfer, vol. 42, no. 19, pp. 3609-3618, 1999.

[80] M. K. Alkam and M. A. Al-Nimr, "Transient non-Darcian forced convection flow in a pipe partially filled with a porous material," International Journal of Heat and Mass Transfer, vol. 41, no. 2, pp. 347-356, 1998.

[81] M. A. Al-Nimr and M. K. Alkam, "Unsteady non-Darcian fluid flow in parallel-plates channels partially filled with porous materials," Heat and Mass Transfer, vol. 33, no. 4, pp. 315-318, 1998.

[82] M. A. Al-Nimr and M. K. Alkam, "A modified tubeless solar collector partially filled with porous substrate," Renewable Energy, vol. 13, no. 2, pp. 165-173, 1998.

[83] M. K. Alkam, M. A. Al-Nimr, and M. O. Hamdan, "On forced convection in channels partially filled with porous substrates," Heat and Mass Transfer, vol. 38, no. 4-5, pp. 337342, 2002.

[84] M. O. Hamdan, M. A. Al-Nimr, and M. K. Alkam, "Enhancing forced convection by inserting porous substrate in the core of a parallel-plate channel," International Journal of Numerical Methods for Heat and Fluid Flow, vol. 10, no. 5, pp. 502-517, 2000.

[85] M. K. Alkam and M. A. Al-Nimr, "Solar collectors with tubes partially filled with porous substrates," Journal of Solar Energy Engineering, vol. 121, no. 1, pp. 20-24, 1999.
[86] J. L. Lage and A. Narasimhan, "Porous media enhanced forced convection fundamentals and applications," in Handbook of Porous Media, K. Vafai and H. A. Hadim, Eds., Marcel Dekker, New York, NY, USA, 2000.

[87] H. R. P. G. Darcy, "Les Fontaines Publiques de la volle de Dijon," Vector Dalmont, Paris, France, 1856.

[88] H. C. Brinkman, "A calculation of the viscous force exerted by a flowing fluid on a dense swarm of particles," Applied Scientific Research, vol. 1, no. 1, pp. 27-34, 1949.

[89] H. C. Brinkman, "On the permeability of media consisting of closely packed porous particles," Applied Scientific Research, vol. 1, no. 1, pp. 81-86, 1949.

[90] J. Bear and Y. Bachmat, Introduction to Modeling of Transport Phenomena in Porous Media, Kluwer Academic Publishers, Dodrecht, The Netherlands, 1990.

[91] K. Vafai and C. L. Tien, "Boundary and inertia effects on flow and heat transfer in porous media," International Journal of Heat and Mass Transfer, vol. 24, no. 2, pp. 195-203, 1981.

[92] A. Amiri and K. Vafai, "Transient analysis of incompressible flow through a packed bed," International Journal of Heat and Mass Transfer, vol. 41, no. 24, pp. 4259-4279, 1998.

[93] B. Alazmi and K. Vafai, "Constant wall heat flux boundary conditions in porous media under local thermal nonequilibrium conditions," International Journal of Heat and Mass Transfer, vol. 45, no. 15, pp. 3071-3087, 2002.

[94] A.-R. A. Khaled and K. Vafai, "The role of porous media in modeling flow and heat transfer in biological tissues," International Journal of Heat and Mass Transfer, vol. 46, no. 26, pp. 4989-5003, 2003.

[95] K. Khanafer and K. Vafai, "Isothermal surface production and regulation for high heat flux applications utilizing porous inserts," International Journal of Heat and Mass Transfer, vol. 44, no. 15, pp. 2933-2947, 2001.

[96] A. Marafie and K. Vafai, "Analysis of non-Darcian effects on temperature differentials in porous media," International Journal of Heat and Mass Transfer, vol. 44, no. 23, pp. 44014411, 2001.

[97] C. W. Sohn and M. M. Chen, "Microconvective thermal conductivity in disperse two-phase mixtures as observed in a low velocity couette flow experiment," Journal of Heat Transfer, vol. 103, no. 1, pp. 47-51, 1981.

[98] A. S. Ahuja, "Augmentation of heat transport in laminar flow of polystyrene suspensions. I. Experiments and results," Journal of Applied Physics, vol. 46, no. 8, pp. 3408-3416, 1975.

[99] A. S. Ahuja, "Augmentation of heat transport in laminar flow of polystyrene suspensions. II. Analysis of the data," Journal of Applied Physics, vol. 46, no. 8, pp. 3417-3425, 1975.

[100] G. Hetsroni and R. Rozenblit, "Heat transfer to a liquid-solid mixture in a flume," International Journal of Multiphase Flow, vol. 20, no. 4, pp. 671-689, 1994.

[101] S. U. S. Choi, "Enhancing thermal conductivity of fluids with nanoparticles," in Developments Applications of NonNewtonian Flows, D. A. Siginer and H. P. Wang, Eds., vol. 231/MD-vol. 66, pp. 99-105, ASME, New York, NY, USA, 1995.

[102] P. Garg, J. L. Alvarado, C. Marsh, T. A. Carlson, D. A. Kessler, and K. Annamalai, "An experimental study on the effect of ultrasonication on viscosity and heat transfer performance of multi-wall carbon nanotube-based aqueous nanofluids," International Journal of Heat and Mass Transfer, vol. 52, no. 21-22, pp. 5090-5101, 2009. 
[103] H. Masuda, A. Ebata, K. Teramae, and N. Hishinuma, "Alteration of thermal conductivity and viscosity of liquid by dispersing ultra-fine particles (dispersion of $\mathrm{c}-\mathrm{Al}_{2} \mathrm{O}_{3}, \mathrm{SiO}_{2}$ and $\mathrm{TiO}_{2}$ ultra-fine particles," Netsu Bussei, vol. 4, pp. 227233, 1993.

[104] J. A. Eastman, S. U. S. Choi, S. Li, L. J. Thompson, and S. Lee, "Enhanced thermal conductivity through the development of nanofluids," in Proceedings of the Materials Research Society (MRS '96), Boston, Mass, USA, 1996.

[105] S. Lee, S. U.-S. Choi, S. Li, and J. A. Eastman, "Measuring thermal conductivity of fluids containing oxide nanoparticles," Journal of Heat Transfer, vol. 121, no. 2, pp. 280-288, 1999.

[106] Y. M. Xuan and Q. Li, "Heat transfer enhancement of nanofluids," International Journal of Heat Fluid Flow, vol. 21, pp. 58-64, 2000.

[107] P. Keblinski, S. R. Phillpot, S. U. S. Choi, and J. A. Eastman, "Mechanisms of heat flow in suspensions of nano-sized particles (nanofluids)," International Journal of Heat and Mass Transfer, vol. 45, no. 4, pp. 855-863, 2001.

[108] H. Xie, J. Wang, T. Xi, Y. Liu, F. Ai, and Q. Wu, "Thermal conductivity enhancement of suspensions containing nanosized alumina particles," Journal of Applied Physics, vol. 91, no. 7, pp. 4568-4572, 2002.

[109] X. Wang, X. Xu, and S. U. S. Choi, "Thermal conductivity of nanoparticle-fluid mixture," Journal of Thermophysics and Heat Transfer, vol. 13, no. 4, pp. 474-480, 1999.

[110] B.-X. Wang, L.-P. Zhou, and X.-F. Peng, "A fractal model for predicting the effective thermal conductivity of liquid with suspension of nanoparticles," International Journal of Heat and Mass Transfer, vol. 46, no. 14, pp. 2665-2672, 2003.

[111] D. Wen and Y. Ding, "Effective thermal conductivity of aqueous suspensions of carbon nanotubes (carbon nanotube nanofluids)," Journal of Thermophysics and Heat Transfer, vol. 18, no. 4, pp. 481-485, 2004.

[112] S. K. Das, N. Putra, and W. Roetzel, "Pool boiling characteristics of nano-fluids," International Journal of Heat and Mass Transfer, vol. 46, no. 5, pp. 851-862, 2003.

[113] S. K. Das, N. Putra, and W. Roetzel, "Pool boiling of nanofluids on horizontal narrow tubes," International Journal of Multiphase Flow, vol. 29, no. 8, pp. 1237-1247, 2003.

[114] S. K. Das, N. Putra, P. Thiesen, and W. Roetzel, "Temperature dependence of thermal conductivity enhancement for nanofluids," Journal of Heat Transfer, vol. 125, no. 4, pp. 567574, 2003.

[115] S. M. You, J. H. Kim, and K. H. Kim, "Effect of nanoparticles on critical heat flux of water in pool boiling heat transfer," Applied Physics Letters, vol. 83, no. 16, pp. 3374-3376, 2003.

[116] P. Vassallo, R. Kumar, and S. D’Amico, "Pool boiling heat transfer experiments in silica-water nano-fluids," International Journal of Heat and Mass Transfer, vol. 47, no. 2, pp. 407-411, 2004.

[117] D. Wen and Y. Ding, "Experimental investigation into the pool boiling heat transfer of aqueous based $\gamma$-alumina nanofluids," Journal of Nanoparticle Research, vol. 7, no. 2-3, pp. 265-274, 2005.

[118] S. Lee and S. U. S. Choi, "Application of metallic nanoparticle suspensions in advanced cooling systems," in Proceedings of the International Mechanical Engineering Congress and Exhibition, Atlanta, Ga, USA, 1996.

[119] B. C. Pak and Y. I. Cho, "Hydrodynamic and heat transfer study of dispersed fluids with submicron metallic oxide particles," Experimental Heat Transfer, vol. 11, no. 2, pp. 151$170,1998$.
[120] Y. Xuan and W. Roetzel, "Conceptions for heat transfer correlation of nanofluids," International Journal of Heat and Mass Transfer, vol. 43, no. 19, pp. 3701-3707, 2000.

[121] Q. Li and Y. Xuan, "Convective heat transfer and flow characteristics of $\mathrm{Cu}$-water nanofluid," Science in China E, vol. 45, no. 4, pp. 408-416, 2002.

[122] Y. Xuan and Q. Li, "Investigation on convective heat transfer and flow features of nanofluids," Journal of Heat Transfer, vol. 125, no. 1, pp. 151-155, 2003.

[123] K. Khanafer, K. Vafai, and M. Lightstone, "Buoyancy-driven heat transfer enhancement in a two-dimensional enclosure utilizing nanofluids," International Journal of Heat and Mass Transfer, vol. 46, no. 19, pp. 3639-3653, 2003.

[124] D. Wen and Y. Ding, "Experimental investigation into convective heat transfer of nanofluids at the entrance region under laminar flow conditions," International Journal of Heat and Mass Transfer, vol. 47, no. 24, pp. 5181-5188, 2004.

[125] J. A. Eastman, S. U. S. Choi, S. Li, W. Yu, and L. J. Thompson, "Anomalously increased effective thermal conductivities of ethylene glycol-based nanofluids containing copper nanoparticles," Applied Physics Letters, vol. 78, no. 6, pp. 718-720, 2001.

[126] F. J. Wasp, Solid-Liquid Slurry Pipeline Transportation, Trans Tech, Berlin, Germany, 1977.

[127] M.-S. Liu, M. C.-C. Lin, C. Y. Tsai, and C.-C. Wang, "Enhancement of thermal conductivity with $\mathrm{Cu}$ for nanofluids using chemical reduction method," International Journal of Heat and Mass Transfer, vol. 49, no. 17-18, pp. 3028-3033, 2006.

[128] W. Yu and S. U. S. Choi, "The role of interfacial layers in the enhanced thermal conductivity of nanofluids: a renovated Maxwell model," Journal of Nanoparticle Research, vol. 5, no. 1-2, pp. 167-171, 2003.

[129] J. Koo and C. Kleinstreuer, "A new thermal conductivity model for nanofluids," Journal of Nanoparticle Research, vol. 6, no. 6, pp. 577-588, 2004.

[130] J. Koo and C. Kleinstreuer, "Laminar nanofluid flow in microheat-sinks," International Journal of Heat and Mass Transfer, vol. 48, no. 13, pp. 2652-2661, 2005.

[131] R. L. Hamilton and O. K. Crosser, "Thermal conductivity of heterogeneous two-component systems," Industrial and Engineering Chemistry Fundamentals, vol. 1, no. 3, pp. 187$191,1962$.

[132] D. A. G. Bruggemen, "Berechnung verschiedener physikalischer Konstanten von heterogenen Substanzen, I. Dielektrizitatskonstanten und Leitfahigkeiten der Mischkorper aus isotropen substanzen," Annalen der Physik, vol. 24, pp. 636679, 1935.

[133] S. M. S. Murshed, K. C. Leong, and C. Yang, "Enhanced thermal conductivity of $\mathrm{TiO}_{2}$ - water based nanofluids," International Journal of Thermal Sciences, vol. 44, no. 4, pp. 367-373, 2005.

[134] Q. Xue and W.-M. Xu, "A model of thermal conductivity of nanofluids with interfacial shells," Materials Chemistry and Physics, vol. 90, no. 2-3, pp. 298-301, 2005.

[135] C. H. Chon, K. D. Kihm, S. P. Lee, and S. U. S. Choi, "Empirical correlation finding the role of temperature and particle size for nanofluid $\left(\mathrm{Al}_{2} \mathrm{O}_{3}\right)$ thermal conductivity enhancement," Applied Physics Letters, vol. 87, no. 15, Article ID 153107, 3 pages, 2005.

[136] R. Prasher, P. Bhattacharya, and P. E. Phelan, "Brownianmotion-based convective-conductive model for the effective thermal conductivity of nanofluids," Journal of Heat Transfer, vol. 128 , no. 6, pp. 588-595, 2006. 
[137] S. P. Jang and S. U. S. Choi, "Effects of various parameters on nanofluid thermal conductivity," Journal of Heat Transfer, vol. 129, no. 5, pp. 617-623, 2007.

[138] X.-Q. Wang and A. S. Mujumdar, "Heat transfer characteristics of nanofluids: a review," International Journal of Thermal Sciences, vol. 46, no. 1, pp. 1-19, 2007.

[139] R. S. Vajjha and D. K. Das, "Experimental determination of thermal conductivity of three nanofluids and development of new correlations," International Journal of Heat and Mass Transfer, vol. 52, no. 21-22, pp. 4675-4682, 2009.

[140] J. Buongiorno, "Convective transport in nanofluids," Journal of Heat Transfer, vol. 128, no. 3, pp. 240-250, 2006.

[141] A. Mokmeli and M. Saffar-Avval, "Prediction of nanofluid convective heat transfer using the dispersion model," International Journal of Thermal Sciences, vol. 49, no. 3, pp. 471-478, 2010.

[142] E. Pfautsch, Forced convection in nanofluids over a flat plate, M.S. thesis, Faculty of the Graduate School, University of Missouri, 2008.

[143] T. P. Cotter, “Theory of heat pipes," Tech. Rep. La-3246- MS, Los Alamos, Science Laboratory, 1965.

[144] L. L. Vasiliev, "Heat pipes in modern heat exchangers," Applied Thermal Engineering, vol. 25, no. 1, pp. 1-19, 2005.

[145] M. Shafahi, V. Bianco, K. Vafai, and O. Manca, "An investigation of the thermal performance of cylindrical heat pipes using nanofluids," International Journal of Heat and Mass Transfer, vol. 53, no. 1-3, pp. 376-383, 2010.

[146] Y. H. Yau and M. Ahmadzadehtalatapeh, "A review on the application of horizontal heat pipe heat exchangers in air conditioning systems in the tropics," Applied Thermal Engineering, vol. 30, no. 2-3, pp. 77-84, 2010.

[147] T. P. Cotter, "Principles and prospects of micro-heat pipe," in Proceedings of the 5th International Heat Pipe Conference, vol. 4, pp. 328-332, Tsukuba, Japan, 1984.

[148] L. L. Vasiliev, "Micro and miniature heat pipes-electronic component coolers," Applied Thermal Engineering, vol. 28, no. 4, pp. 266-273, 2008.

[149] C. B. Sobhan, R. L. Rag, and G. P. Peterson, "A review and comparative study of the investigations on micro heat pipes," International Journal of Energy Research, vol. 31, no. 6-7, pp. 664-688, 2007.

[150] A. F. Khadrawi and M. A. Al-Nimr, "A simplistic way for cooling an intermittent operating internal combustion engine," International Journal of Heat and Technology, vol. 26, no. 2, pp. 163-166, 2008.

[151] K. Vafai and A.-R. A. Khaled, "Analysis of flexible microchannel heat sink systems," International Journal of Heat and Mass Transfer, vol. 48, no. 9, pp. 1739-1746, 2005.

[152] A.-R. A. Khaled, "Analysis of heat transfer inside flexible thinfilm channels with nonuniform height distributions," Journal of Heat Transfer, vol. 129, no. 3, pp. 401-404, 2007.

[153] A.-R. A. Khaled and K. Vafai, "Analysis of thermally expandable flexible fluidic thin-film channels," Journal of Heat Transfer, vol. 129, no. 7, pp. 813-818, 2007.

[154] T. J. Young and K. Vafai, "Convective cooling of a heated obstacle in a channel," International Journal of Heat and Mass Transfer, vol. 41, no. 20, pp. 3131-3148, 1998.

[155] S. Acharya, R. G. Hibbs, Y. Chen, and D. E. Nikitopoulos, "Mass/heat transfer in a ribbed passage with cylindrical vortex generators: the effect of generator-rib spacing," Journal of Heat Transfer, vol. 122, no. 4, pp. 641-652, 2000.
[156] C.-N. Lin and J.-Y. Jang, "Conjugate heat transfer and fluid flow analysis in fin-tube heat exchangers with wave-type vortex generators," Journal of Enhanced Heat Transfer, vol. 9, no. 3-4, pp. 123-136, 2002.

[157] S. Tiwari, P. L. N. Prasad, and G. Biswas, "A numerical study of heat transfer in fin-tube heat exchangers using winglettype vortex generators in common-flow down configuration," Progress in Computational Fluid Dynamics, vol. 3, no. 1, pp. 32-41, 2003.

[158] F. Dupont, C. Gabillet, and P. Bot, "Experimental study of the flow in a compact heat exchanger channel with embossedtype vortex generators," Journal of Fluids Engineering, vol. 125, no. 4, pp. 701-709, 2003.

[159] J. E. O’Brien, M. S. Sohal, and P. C. Wallstedt, "Local heat transfer and pressure drop for finned-tube heat exchangers using oval tubes and vortex generators," Journal of Heat Transfer, vol. 126, no. 5, pp. 826-835, 2004.

[160] Y.-L. Tsay, T. S. Chang, and J. C. Cheng, "Heat transfer enhancement of backward-facing step flow in a channel by using baffle installation on the channel wall," Acta Mechanica, vol. 174, no. 1-2, pp. 63-76, 2005.

[161] A. Joardar and A. M. Jacobi, "Impact of leading edge deltawing vortex generators on the thermal performance of a flat tube, louvered-fin compact heat exchanger," International Journal of Heat and Mass Transfer, vol. 48, no. 8, pp. 14801493, 2005.

[162] M. H. Nasiruddin and K. Siddiqui, "Heat transfer augmentation in a heat exchanger tube using a baffle," International Journal of Heat and Fluid Flow, vol. 28, no. 2, pp. 318-328, 2007.

[163] M. J. Lawson and K. A. Thole, "Heat transfer augmentation along the tube wall of a louvered fin heat exchanger using practical delta winglets," International Journal of Heat and Mass Transfer, vol. 51, no. 9-10, pp. 2346-2360, 2008.

[164] L. H. Tang, M. Zeng, and Q. W. Wang, "Experimental and numerical investigation on air-side performance of fin-andtube heat exchangers with various fin patterns," Experimental Thermal and Fluid Science, vol. 33, no. 5, pp. 818-827, 2009.

[165] E. M. Sparrow, J. E. Niethammer, and A. Chaboki, "Heat transfer and pressure drop characteristics of arrays of rectangular modules encountered in electronic equipment," International Journal of Heat and Mass Transfer, vol. 25, no. 7, pp. 961-973, 1982.

[166] E. M. Sparrow, A. A. Yanezmoreno, and D. R. Otis Jr., "Convective heat transfer response to height differences in an array of block-like electronic components," International Journal of Heat and Mass Transfer, vol. 27, no. 3, pp. 469-473, 1984.

[167] Y.-M. Chen and J.-M. Ting, "Ultra high thermal conductivity polymer composites," Carbon, vol. 40, no. 3, pp. 359-362, 2002.

[168] M. Meis, F. Varas, A. Velázquez, and J. M. Vega, "Heat transfer enhancement in micro-channels caused by vortex promoters," International Journal of Heat and Mass Transfer, vol. 53, no. 1-3, pp. 29-40, 2010.

[169] L. Sheik Ismail, R. Velraj, and C. Ranganayakulu, "Studies on pumping power in terms of pressure drop and heat transfer characteristics of compact plate-fin heat exchangers-a review," Renewable and Sustainable Energy Reviews, vol. 14, no. 1, pp. 478-485, 2010.

[170] S. Eiamsa-ard, K. Wongcharee, P. Eiamsa-ard, and C. Thianpong, "Heat transfer enhancement in a tube using deltawinglet twisted tape inserts," Applied Thermal Engineering, vol. 30 , no. 4 , pp. $310-318,2010$. 
[171] G. Tanda, "Natural convective heat transfer in vertical channels with low-thermal-conductivity ribs," International Journal of Heat and Fluid Flow, vol. 29, no. 5, pp. 1319-1325, 2008.

[172] A. Saidi and B. Sundén, "A numerical investigation of heat transfer enhancement in offset strip fin heat exchangers in self-sustained oscillatory flows," International Journal of Numerical Methods for Heat and Fluid Flow, vol. 11, no. 7, pp. 699-717, 2001.

[173] B. A. Jubran, S. A. Swiety, and M. A. Hamdan, "Convective heat transfer and pressure drop characteristics of various array configurations to simulate the cooling of electronic modules," International Journal of Heat and Mass Transfer, vol. 39, no. 16, pp. 3519-3529, 1996.

[174] T. J. Young and K. Vafai, "Convective flow and heat transfer in a channel containing multiple heated obstacles," International Journal of Heat and Mass Transfer, vol. 41, no. 21, pp. 3279-3298, 1998.

[175] M. K. Alkam, M. A. Al-Nimr, and M. O. Hamdan, "Enhancing heat transfer in parallel-plate channels by using porous inserts," International Journal of Heat and Mass Transfer, vol. 44, no. 5, pp. 931-938, 2001.

[176] J. Ting and M. L. Lake, "Vapor grown carbon fiber reinforced aluminum composites with very high thermal conductivity," Journal of Materials Research, vol. 10, no. 2, pp. 247-250, 1995.

[177] K. Naito, J.-M. Yang, Y. Xu, and Y. Kagawa, "Enhancing the thermal conductivity of polyacrylonitrile- and pitchbased carbon fibers by grafting carbon nanotubes on them," Carbon, vol. 48, no. 6, pp. 1849-1857, 2010. 

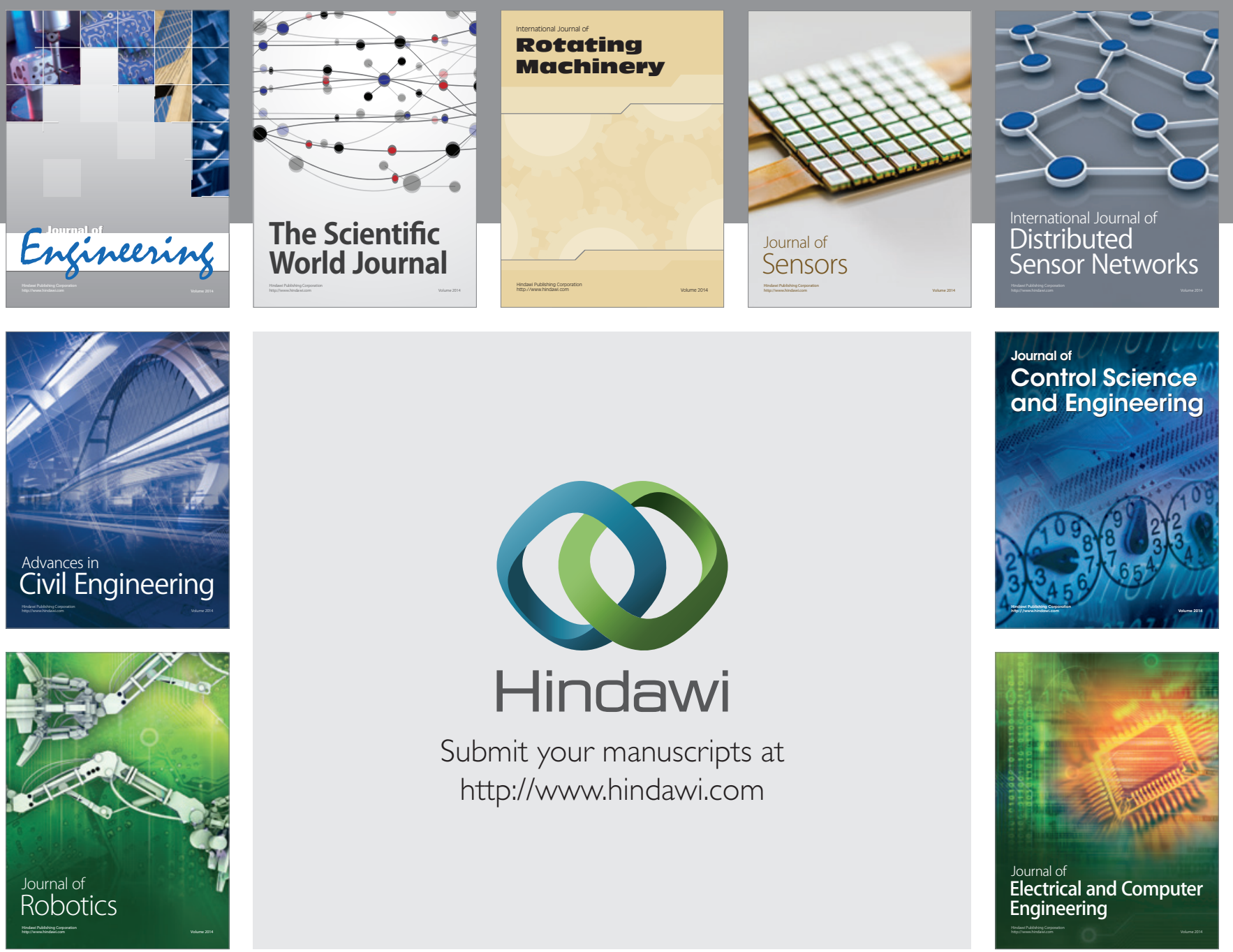

Submit your manuscripts at

http://www.hindawi.com
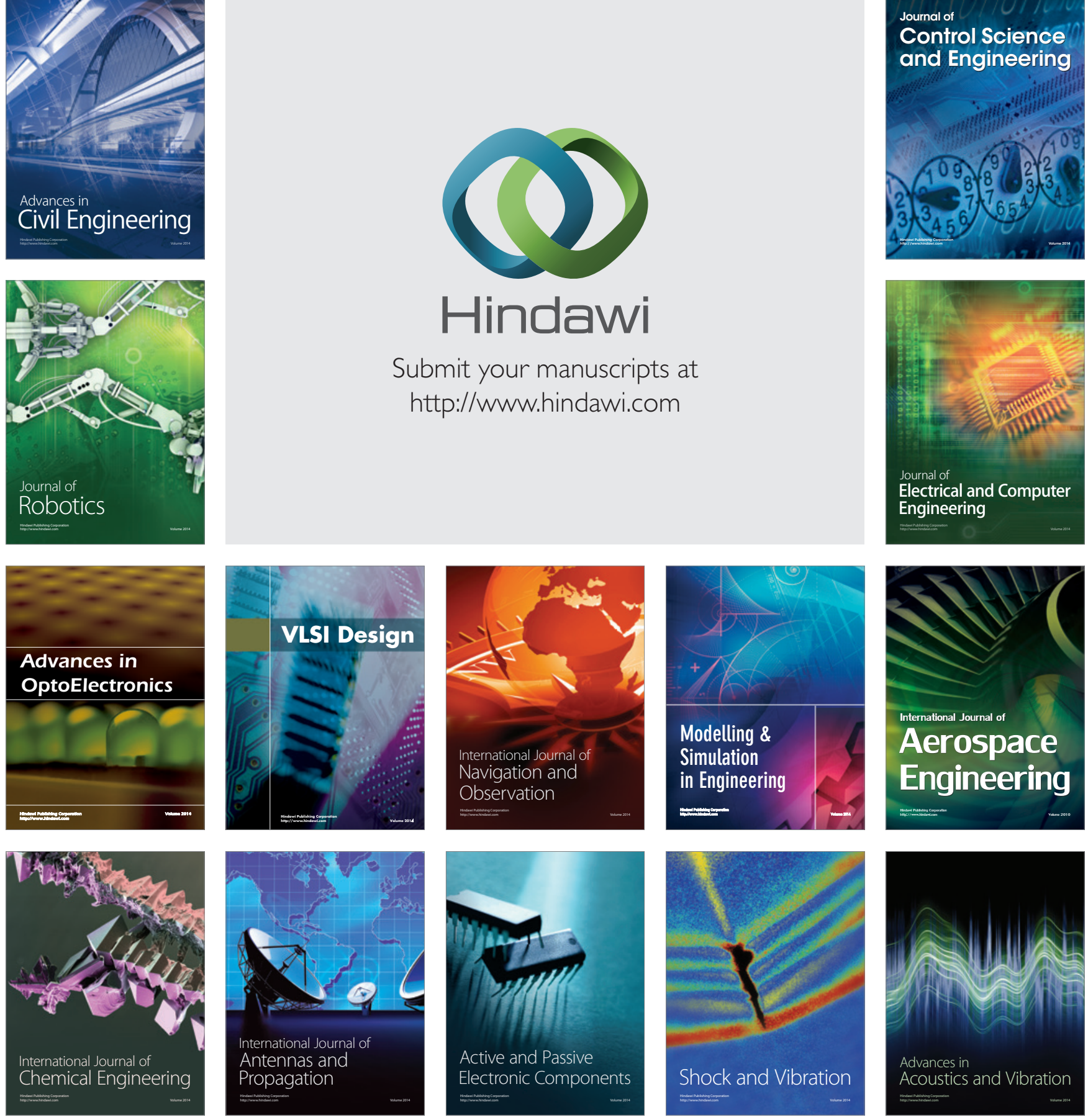